\title{
Quantitative investigation of micro slip and localization in polycrystalline materials under uniaxial tension
}

DOI:

10.1016/j.ijplas.2018.04.014

\section{Document Version}

Accepted author manuscript

Link to publication record in Manchester Research Explorer

\section{Citation for published version (APA):}

Zhang, Z., Lunt, D., Abdolvand, H., Wilkinson, A. J., Preuss, M., \& Dunne, F. P. E. (2018). Quantitative investigation of micro slip and localization in polycrystalline materials under uniaxial tension. International Journal of Plasticity. https://doi.org/10.1016/.i.jplas.2018.04.014

\section{Published in:}

International Journal of Plasticity

\section{Citing this paper}

Please note that where the full-text provided on Manchester Research Explorer is the Author Accepted Manuscript or Proof version this may differ from the final Published version. If citing, it is advised that you check and use the publisher's definitive version.

\section{General rights}

Copyright and moral rights for the publications made accessible in the Research Explorer are retained by the authors and/or other copyright owners and it is a condition of accessing publications that users recognise and abide by the legal requirements associated with these rights.

\section{Takedown policy}

If you believe that this document breaches copyright please refer to the University of Manchester's Takedown Procedures [http://man.ac.uk/04Y6Bo] or contact uml.scholarlycommunications@manchester.ac.uk providing relevant details, so we can investigate your claim.

\section{OPEN ACCESS}




\title{
Quantitative investigation of micro slip and localization in polycrystalline materials under uniaxial tension
}

Zhen Zhang ${ }^{\mathrm{a}}$, David Lunt ${ }^{\mathrm{b}}$, Hamidreza Abdolvand ${ }^{\mathrm{c}, \mathrm{d}}$, Angus J. Wilkinson ${ }^{\mathrm{c}}$, Michael Preuss ${ }^{\mathrm{b}}$, Fionn P.E. Dunne ${ }^{\mathrm{a}}$

aDepartment of Materials, Imperial College, London, Prince Consort Road, SW7 2AZ, United Kingdom

${ }^{b}$ BP International Centre for Advanced Materials, School of Materials, University of Manchester, M13 9PL, United Kingdom

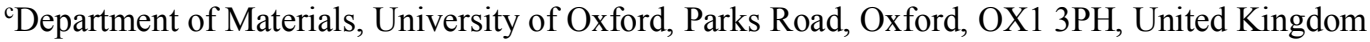

${ }^{\mathrm{d} D e p a r t m e n t}$ of Mechanical \& Materials Engineering, Spencer Engineering Building, Western University, N6A 5B9, Canada

\begin{abstract}
Micro slip activation and localization in Ti-6Al-4V deformed in tension have been examined quantitatively using high-resolution (HR) digital image correlation (DIC), HR-electron backscatter diffraction (EBSD) and crystal plasticity finite element modelling. The measured polycrystal slip, strain, lattice rotation and geometrically necessary dislocation (GND) density distributions are generally well captured by the a priori crystal plasticity model based on the rate-sensitive properties of a-titanium. An overall slip trace analysis showed over $80 \%$ agreement between HR-DIC and crystal plasticity modelling of the primary slip activation. The texture beneath the characterised free-surface has been found to affect the local slip, stress distribution, lattice curvature and GND density and three texture variations have been considered. Grain-level slip trace analysis shows that the crystal plasticity modelling can capture single (straight) slip, multiple slip activation and complex wavy slip. The latter has been found to result from the interaction of independently activated basal and prismatic slip systems with common slip direction. Initial inter-granular misorientations greater than about $5^{\circ}$ have been shown to influence the subsequent micromechanical grain behaviour including slip, lattice rotation and GND density. This work contributes to the understanding of slip localization and load shedding in dwell fatigue in polycrystalline hexagonal materials.
\end{abstract}

Keyword: high resolution EBSD, high resolution DIC, crystal plasticity finite element modelling, slip localization, straight and wavy slip traces, grain misorientation, HCP polycrystals 


\section{Introduction}

Crystalline materials such as hexagonal alloys often comprise multi-phase microstructures with complex morphologies, which is an intended result of alloy composition and material processing route (Attallah et al., 2009; Lütjering and Williams, 2007). Due to the elastic and plastic response of a crystal, local plasticity within the material is highly anisotropic (Abdolvand et al., 2018; Britton et al., 2010a; Kasemer et al., 2017). Under the external loading introduced by component operation, local shear stresses can be substantial and drive dislocation motion along slip planes and across differing phases. The deformation is accommodated by the most easily activated slip systems, which may possess differing rate dependence. The collective slip activities on the slip planes in individual grains lead to the plasticity developed in the material. Time sensitive stress redistribution may be established among grains. For example, the combination of soft grains well orientated for slip versus elastically deformed hard grains can cause notable load shedding, which has been argued to be a contributory factor in facet nucleation in some titanium alloys (Dunne and Rugg, 2008). Hence, it is imperative to quantify microscale slip and deformation localization through well calibrated micro-testing techniques and reliable computational methods.

Slip localization in polycrystalline materials has been reported by using high resolution digital image correlation (HR-DIC) (Di Gioacchino and da Fonseca, 2013), high resolution electron backscattered diffraction (HR-EBSD) (Britton and Wilkinson, 2012; Wilkinson et al., 2006a) and crystal plasticity modelling (Guery et al., 2016; Tasan et al., 2014). The local deformation, in the form of slip bands along highly stressed and well aligned slip systems, may be captured by cross-correlation of patterns of distributed nano-particles on the surface of deformed samples compared to the previously undeformed state. Therefore, the in-plane displacement and subsequently the strain field can be calculated (Vendroux and Knauss, 1998). In this way, the HR-DIC technique has been utilised extensively in studying the 
microscale deformation fields (Echlin et al., 2016; Lunt et al., 2017b), the role of aging treatment (Lunt et al., 2018), and $\alpha_{2}$ precipitation (Lunt et al., 2017a) in strain localization of dual phase Ti-6Al-4V. It has also been applied to investigate single, polycrystal and polycrystalline (with inclusions) nickel-based superalloys (Guan et al., 2017; Jiang et al., 2016; Zhang et al., 2015b), as well as ferritic-martensitic dual phase steel (Tasan et al., 2014) and austenitic stainless steel (A316LN) (Guery et al., 2016). In addition, lattice distortions from elastic strain variations and lattice rotations within the sample cause small shifts in the positions of zone axes in the EBSD patterns. The shifts at regions of interest across the EBSD patterns may be utilised to determine directly the "relative" elastic deformation tensor containing information on the arbitrary strain and rotation. The relative variation in the entire strain and rotation tensor can be obtained from cross correlating Kikuchi patterns (Wilkinson et al., 2006a, b) with respect to those acquired at a reference point, which is a region of uniform, bulk crystallographic orientation. The cross-correlation-based analysis on the EBSD patterns allows rotations to be measured at much greater sensitivity of $\sim 0.01^{\circ}$ compared to $1-0.5^{\circ}$ when using the "conventional" Hough transform-based analysis (Wilkinson et al., 2006a). In this way, the HR-EBSD measurements of spatial variation in crystal orientation can be used to recover absolute GND densities utilising Nye's framework (El-Dasher et al., 2003; Nye, 1953; Sun et al., 2000; Wilkinson and Randman, 2010).

Crystal plasticity modelling is now commonly used to assess and interpret both HR-DIC measurements of total strain fields as well as the elastic strain and GND fields from HREBSD, and several research groups have reported work using these techniques in recent years (Tasan et al., 2014; Zhang et al., 2014). The resolution of strain measurements in HR-DIC ( $60-250 \mathrm{~nm}$ ) is sufficiently good such that individual slip traces from plastic deformation can be clearly captured (Di Gioacchino and da Fonseca, 2013; Lunt et al., 2017b). HR-DIC and crystal plasticity modelling have been used to investigate the strain localization in dual phase 
steel (Tasan et al., 2014) where it was found that plasticity typically initiates within "hot zones", where there are larger ferritic grains and a lower local martensite fraction than the bulk material. DIC measurements and crystal plasticity modelling have been utilised to study the slip activity in polycrystalline austenitic stainless steel (A316LN) (Guery et al., 2016). Good agreement was achieved in the major slip system evaluation between experimental observation and computations. Quantitative comparisons between shear strain maps for the simulated and experimental results have been obtained in a sample with a large average grain size $(70 \mu \mathrm{m})$ and in a sample with a low volume fraction of small grains down to $5 \mu \mathrm{m}$ coexisting with larger grains up to $200 \mu \mathrm{m}$. Crystal plasticity modelling has also been combined with HR-EBSD to study twin tip local deformations (Abdolvand and Wilkinson, 2016a, b; Guo et al., 2017). The strain field measured by HR-EBSD is based on a single EBSD pattern such that the strain in a grain is always measured relative to the reference point within that grain. However, considering the reference points consistently as in HR-EBSD, it is interesting to note that the grain level strain fields are well-represented by crystal plasticity modelling (Zhang et al., 2014). Since the calculation of GNDs is based on the variation in lattice rotation, absolute values of GNDs can be recovered by HR-EBSD without having to consider a reference point. In addition, the comparison between crystal plasticity modelling and the HR-EBSD results are confined to the strain and lattice rotation fields on selected and limited grains. There is limited open data on the quantitative comparison of polycrystalline GND densities and those from crystal level modelling techniques. This demands highfidelity modelling tools that are well calibrated against appropriately designed microscale experiments.

The parallel use of HR-DIC and HR-EBSD in conjunction with computational crystal plasticity modelling has rarely been reported when studying the micromechanical behaviour of polycrystals. It remains a question whether these techniques can provide a quantitative and 
mechanistic analysis of challenging slip activities involving planar and wavy slip lines in polycrystals. Interestingly, clear micro slip bands, containing mostly planar traces, have been observed in polycrystalline stainless steel (Di Gioacchino and da Fonseca, 2015), and nickelbased superalloy (Stinville et al., 2015). In contrast, straight and wavy slip lines have been reported in titanium alloys. Basal slip lines have been found to be wavy in single crystal $\alpha$ titanium alloys (Akhtar, 1975; Akhtar and Teghtsoonian, 1975; Williams et al., 2002), though the formation mechanism of these wavy slip bands remains elusive. Previously, it has been stated that crystal plasticity modelling cannot capture the microscale deformation state (Di Gioacchino and da Fonseca, 2015). However, recent crystal plasticity modelling seems to be encouraging as it can reproduce the slip traces observed in single crystals (Zhang et al., 2016b) and dual phase titanium alloys (Zhang et al., 2016a). Good predictions have been shown for micro-pillars ( $2 \mu \mathrm{m}$ wide) during elastic-plastic deformation, and for stress relaxation, slip activation in both $\alpha$ and $\beta$-phases, and strain localisation within the $\alpha-\beta$ pillars with differing test strain rates, $\beta$ morphology, and crystal orientations (Zhang et al., 2016a, b). These recent finding have motivated the present study in which slip activities in rate dependent polycrystalline Ti-6Al-4V is investigated by using crystal plasticity modelling to interpret the measurements from both HR-DIC and HR-EBSD techniques.

The subject of this paper is crystal slip localization analysis in a uniaxially tensioned polycrystalline Ti-6Al-4V sample. A comparative study has been conducted using quantitative crystal plasticity modelling and experimental measurements captured using both the HR-DIC and HR-EBSD techniques. The slip activity is examined at both the macroscale and at the grain level by addressing local strain, lattice rotation, and GND densities. Detailed slip analysis is also highlighted in selected individual grains having single, multiple and complex (wavy) slip trace characteristics. Finally, the uncertainties arising in the modelling 
from initial grain level misorientation and polycrystal layers beneath the observed free surface are also discussed.

\section{Experiment and material characterization}

The material in this study is Ti-6Al-4V that was forged and annealed but not rolled, leading to a weakly textured material without significant microtexture development and an average grain size of $\sim 20 \mu \mathrm{m}$. More details regarding the material can be found in (Lunt et al., 2017b) where it is termed no-macrozone condition. To produce a more simplistic microstructure, the material was subjected to a homogenisation heat treatment at $950{ }^{\circ} \mathrm{C}$ for 2 hours followed by slow cooling to generate an equiaxed microstructure. Subsequently, the material was annealed at $700{ }^{\circ} \mathrm{C}$ for 1 hour followed by water quenching to avoid any $\alpha_{2}$ precipitation. Subsequently, flat dogbone tensile samples were electrical discharge machined from the heat-treated block with a $26 \mathrm{~mm}$ gauge length, $3 \mathrm{~mm}$ gauge width and $1 \mathrm{~mm}$ thickness.

\subsection{High Resolution Digital Image Correlation (HR-DIC)}

The sample was prepared for HR-DIC by initial grinding to \#4000 grit paper followed by hand polishing in a solution of 4:1 colloidal silica to hydrogen peroxide for 30 minutes. After polishing, a grid of micro-hardness indents was made on the sample surface to enable easy identification of the region for HR-DIC analysis. The final sample thickness, after polishing, was $0.91 \mathrm{~mm}$. A gold speckle pattern was applied to the surface of the sample using the gold remodelling technique (Di Gioacchino and da Fonseca, 2013), that has subsequently been optimised for HR-DIC studies on titanium alloys (Lunt et al., 2017a; Lunt et al., 2018). Before remodelling, a gold layer of 25-40 nm was deposited onto the sample surface using an Edwards S150B sputter. The sample was then remodelled in a water vaporisation 
environment at $300{ }^{\circ} \mathrm{C}$ for 3 hours to generate a pattern with individual speckles of $60-100$ $\mathrm{nm}$ that have a uniform distribution, as shown from the magnified backscattered electron image (Figure 1).

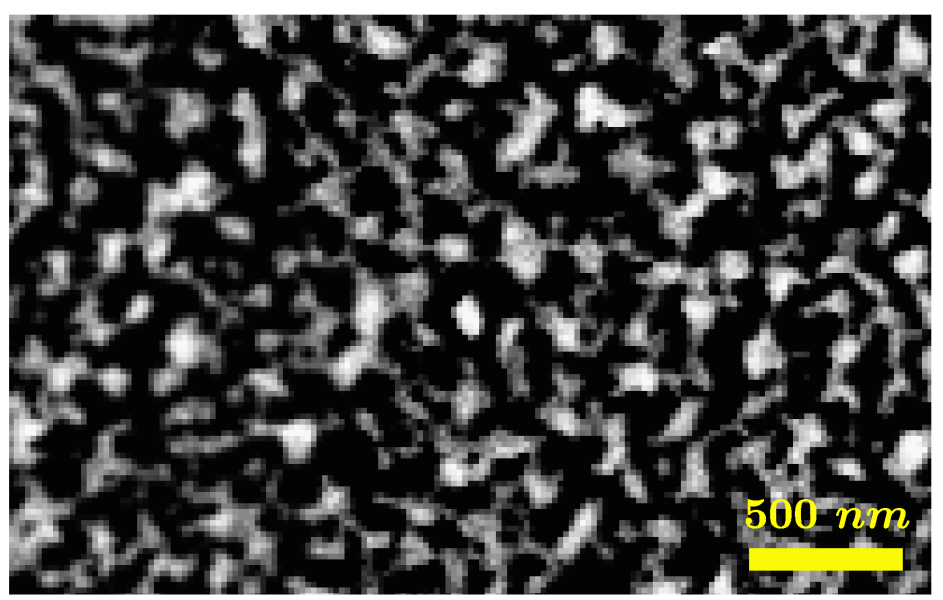

Figure 1 Typical speckle pattern on the sample surface for HR-DIC analysis

The images for HR-DIC were acquired, in backscattered electron mode at $20 \mathrm{kV}$ at a working distance of $\sim 5 \mathrm{~mm}$, using a FEI Quanta 650 Field Emission Gun Scanning Electron Microscope (FEG-SEM). A matrix of 4 x 4 images were acquired at a resolution of $2048 \times$ 1768 pixels $^{2}$ with a single image horizontal field width of $29.6 \mu \mathrm{m}$, equating to a total area for the HRDIC region of $\sim 100 \times 80 \mu \mathrm{m}^{2}$. Each image took 30 seconds to record and was taken with a $10 \mu$ s dwell time. After the initial matrix of images was taken of the undeformed state, the sample was removed from the microscope and placed into a Kammrath-Weiss $5 \mathrm{kN}$ tension-compression microtester where it was deformed in tension to $\sim 3 \%$ applied strain at room temperature at a displacement rate of $0.1 \mathrm{~mm} / \mathrm{min}$ and strain rate of $6.4 \times 10^{-5} \mathrm{~s}^{-1}$. After deformation, the sample was again placed back inside the SEM, and the matrix of images from the same area of the deformed state was recorded.

The main principle behind Digital Image Correlation (DIC) is that it tracks unique features and compares their position between the deformed and undeformed states. The relative 
displacement of these features is used to produce a full-field displacement map that can subsequently be differentiated to calculate the desired strain field. In this study, HR-DIC was performed using the commercially available La Vision's Davis imaging software. Displacement maps were produced from the previously stitched matrix of images and processed using a standard Fast Fourier Transform (FFT) with an interrogation window size of $147 \times 147 \mathrm{~nm}^{2}$ and a $0 \%$ overlap, which has been shown previously to provide an acceptable compromise between spatial resolution and systematic errors associated with rastering (Lunt et al., 2017a).

\subsection{Experiment and sample preparation for HR-EBSD}

The HR-EBSD measurement was carried out on the same area as the HR-DIC was performed. After the HR-DIC measurement, the deformed sample was polished briefly to remove the gold layer. The EBSD scan was performed in a Zeiss MERLIN Field Emission Scanning Electron Microscope (FEG-SEM) with operating condition of $20 \mathrm{keV}$ and a probe current of 15 nA. EBSD patterns were captured using a high resolution Bruker detector at a step size of $0.2 \mu \mathrm{m}$. The exposure time for collecting each $800 \times 600$ pixels $^{2}$ Kikuchi pattern was 200 milliseconds.

Elastic strain and lattice rotations were calculated by cross correlating the collected diffraction patterns (Wilkinson et al., 2006a). This was done by dividing each pattern into 40 subdomains selected within the pattern. Subdomains were cross correlated with those from a reference point (based on the mean value of the measured quantity) that was selected for each grain. The shifts and movements of each pattern were calculated from which the relative elastic lattice rotation and strain were calculated. Since lattice rotations can be large, particularly at stress concentration sites, the remapping method was used herein (Britton and 
Wilkinson, 2012). In this method, rotations calculated from the first pass were used to remap the test pattern intensities into the reference pattern orientation and then with the second pass of cross correlation, elastic strains and rotations were recalculated. The single crystal elastic modulus was used to calculate relative stresses from measured relative elastic strains. The absolute values of geometrically necessary dislocation densities were calculated using spatial variation of measured lattice curvature and the Nye tensor (Nye, 1953). Because there is no unique relationship between lattice curvature and the nine components of the Nye tensor, an L1 minimisation technique based on dislocation energy is utilised as is conventional to give a lower bound on GND density (Britton et al., 2010b).

\subsection{High resolution DIC and EBSD strain calculations}

The total strain field has been calculated from the HR-DIC measured displacement $\mathbf{u}$ given by

$$
\varepsilon=\frac{1}{2}\left(\nabla \mathbf{u}+\nabla \mathbf{u}^{\mathrm{T}}\right)
$$

The shear strain map, if applicable, is typically represented in terms of the maximum effective shear strain $\left(\gamma_{\max }\right)$ (Di Gioacchino and da Fonseca, 2013; Lunt et al., 2017a) as follows:

$$
\gamma_{\max }=\sqrt{\left(\frac{\left(\varepsilon_{x x}-\varepsilon_{y y}\right)}{2}\right)^{2}+\left(\frac{\left(\varepsilon_{x y}+\varepsilon_{y x}\right)}{2}\right)^{2}}
$$

In the HR-EBSD measurements, with knowledge of the elastic deformation gradient $\mathbf{F}^{\mathbf{e}}$, and provided the strains and rigid body rotations are small, the lattice rotation $\omega^{\mathbf{e}}$ may be determined from an additive decomposition as 


$$
\boldsymbol{\omega}^{\mathbf{e}}=\mathbf{F}^{\mathbf{e}}-\mathbf{I}-\boldsymbol{\varepsilon}^{\mathbf{e}}
$$

The elastic strains and lattice rotations are measured from diffraction pattern shifts (Britton and Wilkinson, 2011; Wilkinson et al., 2006a), and the lower bound geometrically necessary dislocation density is solved based on an L1 normalization (Britton et al., 2010b).

\subsection{Slip Trace Analysis}

The likely active slip system for each grain has been determined using the slip trace analysis technique (Bridier et al., 2005; Hémery and Villechaise, 2017; Lunt et al., 2017a). The slip trace angles measured from the HR-DIC are cross correlated with theoretical slip trace angles for all slip systems (prismatic $<\mathrm{a}>$, basal $<\mathrm{a}>$ and pyramidal $<\mathrm{a}>/<\mathrm{c}+\mathrm{a}>$ ) that have been calculated using the average crystal orientation for each grain obtained from the EBSD data. Using this approach, it is not possible to distinguish between pyramidal $<\mathrm{a}>$ and $1^{\text {st }}$ order pyramidal $<\mathrm{c}+\mathrm{a}>$, as the two slip systems give the same slip trace angle projection on the investigated surface. Therefore, they were initially grouped together as pyramidal slip and the Schmid factor was used to distinguish between the two in any further analysis. $\mathrm{A} \pm 5^{\circ}$ angle criterion between the experimental and theoretical slip angle is applied to predict the likely slip mode. In grains where more than one slip mode lies within $\pm 5^{\circ}$, the slip system with the highest apparent Schmid factor has been predicted as the likely slip mode. This does not provide a satisfactory way of determining the active slip mode. However, due to the limited number of grains investigated, it does allow all grains to be considered in the comparison of active slip modes between HR-DIC and CP modelling.

\section{Crystal plasticity modelling framework considering rate dependence}


Rate dependent crystal plasticity with updated lattice rotation is utilised in this study (Zhang et al., 2016a). GND and statistically stored dislocation (SSD) density evolution are considered such that slip activation, spatial reach and distribution of slip, as well as elastic and total strains, and GND densities, may be compared directly with the experimental observations from HR-DIC and HR-EBSD.

\subsection{Kinematics}

The multiplicative elastic-plastic decomposition of the deformation gradient is given by

$$
\mathbf{F}=\mathbf{F}^{\mathrm{e}} \mathbf{F}^{\mathrm{p}}
$$

The plastic deformation gradient evolves at a rate given by

$$
\dot{\mathbf{F}}^{\mathrm{p}}=\mathbf{L}^{\mathrm{P}} \mathbf{F}^{\mathrm{P}}
$$

The plastic velocity gradient $\mathbf{L}^{\mathrm{P}}$ is that associated with plastic flow through a fixed lattice and is given by

$$
\mathbf{L}^{\mathrm{P}}=\sum_{i} \dot{\gamma}^{i} \mathbf{s}^{i} \otimes \mathbf{n}^{i}
$$

where $\mathbf{s}^{i}$ and $\mathbf{n}^{i}$ are updated slip directions and plane normals. The slip rate $\dot{\gamma}$ is given by

$$
\dot{\gamma}^{i}=\rho_{m} v b^{2} \exp \left(-\frac{\Delta \mathrm{F}}{k \mathrm{~T}}\right) \sinh \left[\frac{\left(\tau^{i}-\tau_{c}^{i}\right) \Delta \mathrm{V}}{k \mathrm{~T}}\right]
$$

where $i$ is the active slip system (whether in the $\alpha$ or $\beta$ phase). $\rho_{m}$ is the density of mobile dislocations, $v$ the frequency of attempts of dislocations to jump obstacle energy barriers, $b$ the Burger's vector magnitude, $k$ Boltzman's constant, $\mathrm{T}$ the temperature, $\tau^{i}$ the resolved shear stress for the activated slip system, and $\tau_{c}^{i}$ is the corresponding critical resolved shear stress (CRSS). In particular, $\Delta \mathrm{F}$ is the activation energy, and $\Delta \mathrm{V}$ the corresponding activation 
volume for the thermally activated escape of pinned dislocations, governing the overall rate sensitive deformation (i.e. creep behaviour).

In the crystal modelling, the GNDs and statistically stored dislocations (SSDs) contribute to the critical resolved shear stress and consequently the strain hardening (Ashby, 1970; Devincre et al., 2008; Franciosi et al., 1980; Gao and Huang, 2001; Nye, 1953), as follows

$$
\tau_{\mathrm{c}}=\tau_{\mathrm{c} 0}+G b \sqrt{\rho_{\mathrm{G}}+\rho_{\mathrm{S}}}
$$

The plastic spin term is given by the anti-symmetric part of the plastic velocity gradient

$$
\mathbf{W}^{\mathrm{P}}=\sum_{i} \operatorname{asym}\left(\dot{\gamma}^{i} \mathbf{s}^{i} \otimes \mathbf{n}^{i}\right)
$$

so that the crystal orientation may be updated by the lattice spin, given by

$$
\mathbf{W}^{\mathrm{e}}=\mathbf{W}-\mathbf{W}^{\mathrm{P}}
$$

where the continuum spin $\mathbf{W}$, is given by the antisymmetric part of the total velocity gradient, $\mathbf{L}$

$$
\mathbf{W}=\operatorname{asym}(\mathbf{L})
$$

where $\mathbf{L}=\dot{\mathbf{F}} \mathbf{F}^{-\mathbf{1}}$, and $\mathbf{F}$ is the current deformation gradient.

The rate of crystal plastic deformation is

$$
\mathbf{D}^{\mathrm{P}}=\mathbf{L}^{\mathrm{P}}-\mathbf{W}^{\mathrm{P}}
$$

such that the Jaumann stress rate through Hooke's law is given by

$$
\widehat{\boldsymbol{\sigma}}=\mathbf{C}:\left(\mathbf{D}-\mathbf{D}^{p}\right)
$$


where $\mathbf{C}$ is the anisotropic material tangential stiffness tensor which is updated incrementally. Elastic properties with respect to undeformed material axes are given in Table 1. The deformation rate is

$$
\mathbf{D}=\operatorname{sym}(\mathbf{L})
$$

Finally, the material rate of stress is

$$
\dot{\boldsymbol{\sigma}}=\widehat{\boldsymbol{\sigma}}+\mathbf{W} \boldsymbol{\sigma}-\sigma \mathbf{W}
$$

where $\sigma$ is the current stress state.

This stress increment is updated to determine the appropriate increment of plastic strain such that the consistency condition is satisfied through Newton iteration.

\subsection{The GND and SSD contribution}

The evolution of SSDs is taken simply to be (Zhang et al., 2015c)

$$
\dot{\rho}_{\mathrm{S}}=\gamma^{\prime} \dot{\mathrm{p}}
$$

where $\gamma^{\prime}$ is the coefficient for SSD hardening and the rate of accumulated effective plastic strain is $\dot{\mathrm{p}}=\sqrt{\frac{2}{3} \mathbf{D}^{\mathrm{P}}: \mathbf{D}^{\mathrm{P}}}$.

The GND density is calculated from the gradient of the plastic part of the deformation gradient from a Nye analysis

$$
\sum_{i}^{N_{\mathrm{s}}} \mathbf{b}_{\mathrm{G}}^{i} \otimes \boldsymbol{\rho}_{\mathrm{G}}=\operatorname{curl}\left(\mathbf{F}^{\mathrm{P}}\right)
$$

Nye's dislocation tensor $\Lambda$ is used to compute the components of the GNDs, given by 


$$
\boldsymbol{\Lambda}=\operatorname{curl}\left(\mathbf{F}^{\mathbf{P}}\right)=\sum_{\alpha=1}^{12} \rho_{\mathrm{Gs}}^{\alpha} \mathbf{b}^{\alpha} \otimes \mathbf{s}^{\alpha}+\rho_{\mathrm{Get}}^{\alpha} \mathbf{b}^{\alpha} \otimes \mathbf{t}^{\alpha}+\rho_{\mathrm{Gen}}^{\alpha} \mathbf{b}^{\alpha} \otimes \mathbf{n}^{\alpha}
$$

in which $\rho_{\mathrm{Gs}}^{\alpha}$ are screw dislocation components on slip system $\alpha$ with line vector along the slip direction $\mathbf{s}^{\alpha}$, while $\rho_{\mathrm{Get}}^{\alpha}$ and $\rho_{\mathrm{Gen}}^{\alpha}$ are edge dislocations and their line tangent vectors are parallel to slip normal $\mathbf{n}^{\alpha}$ and $\mathbf{t}^{\alpha}$ respectively where $\mathbf{t}^{\alpha}=\mathbf{s}^{\alpha} \times \mathbf{n}^{\alpha} . \mathbf{b}^{\alpha}$ is the Burgers vector on slip system $\alpha$. In order to compute the independent GND edge and screw components, the above equation can be expressed as:

$$
\mathbf{A} \rho_{\mathrm{G}}=\Lambda
$$

and

$$
\boldsymbol{\rho}_{\mathrm{G}}=\mathbf{A}^{+} \boldsymbol{\Lambda}
$$

where $\mathbf{A}$ is a tensor formulated in accordance with the methodology developed by Cheng and Ghosh (2015) and the matrix of the pseudo-inverse of $\mathbf{A}$ is expressed as $\mathbf{A}^{+}$.

The GND methodology adopted originates from Nye (1953), and is consistent with Cermelli and Gurtin (2001) when elastic lattice strains are small (Clayton, 2010; Gurtin, 2002; Steinmann, 2015). More details of the formation adopted in the current study can be found in Arsenlis and Parks (1999) and Busso et al. (2000).

\section{Results and Discussion}

The current work is a comparative study, using crystal plasticity modelling, HR-DIC and HR-EBSD, of the polycrystalline elastic-plastic response of alloy Ti-6Al-4V with a particular emphasis on slip activity and localization, grain-level slip traces, lattice rotations, strains and GND densities. Discussion includes grain level misorientation, the free surface response (where measurements are made) and the response potentially resulting from the unknown anisotropic polycrystal layer of material beneath the free surface. 


\subsection{Macroscopic response}

As shown in Figure 2, the crystal plasticity modelled region of the sample under investigation is a square with an area of $240 \times 240 \mu \mathrm{m}^{2}$. Crystallographic orientations have been assigned from those measured by EBSD, but at the end of deformation. However, given the applied strain of $3.3 \%$, these orientations are unlikely to be significantly different from those prior to deformation. The out-of-plane depth of the model is taken to be $20 \mu \mathrm{m}$, which is the average grain size of all the grains illustrated in the inverse pole figure in Figure 2(a). This size of modelled region is selected to ensure the centre grain, highlighted by the purple broken line in Figure 2(b), is well represented for the grain level study. In addition, the modelled region, illustrated in Figure 2(b), includes the full scanned area used for HR-DIC $\left(132 \times 86 \mu \mathrm{m}^{2}\right)$ and HR-EBSD $\left(176 \times 120 \mu \mathrm{m}^{2}\right)$. The HR-DIC and HR-EBSD measurements were taken on the same surface of the tested specimen to provide a consistent comparison of experimental full field strain and strain gradient measurements with the crystal plasticity models. As a result, the CPFE model considered contains 176 grains as shown in Figure 2(c).

To facilitate the comparative study of unknown material beneath the measured free surface, a second layer of material with a grain size of $20 \mu \mathrm{m}$ is also considered as in Figure 2(d). The grain shapes are taken to be prismatic along the $\mathrm{z}$ direction; that is, the grain morphology of the sub-layer is identical to the free surface layer, but the sub-layer grain orientations may be specified differently to those for the free surface. This consideration places emphasis on investigating the role of crystallography of the underlying sub-layer of grains on the surface deformation as opposed to the lesser effect of grain morphology. The crystal plasticity model is meshed by 20 noded ABAQUS elements C3D20R. The material parameters used in the model presented are listed in Table 1. With a prior convergence study, the stress-strain curve is found to produce experimentally consistent results when the mesh size reaches $4 \mu \mathrm{m}$ per element, as shown in Figure 2(c). The same mesh has also been 
adopted for the double layer model in Figure 2(d). The crystal orientations of the sub-layer are taken to be random in the double layer model when it is utilised, or as otherwise specified.

Table 1 Slip rule properties for Ti- $6 \mathrm{Al}$ at $20^{\circ} \mathrm{C}^{1}$

\begin{tabular}{|c|c|c|}
\hline \multicolumn{2}{|c|}{ Parameters } & Values \\
\hline \multirow{5}{*}{$\begin{array}{c}\text { Elastic } \\
\text { constants }\end{array}$} & E11 (MPa) & 84745 \\
\hline & E33 (MPa) & 119789 \\
\hline & G13 (MPa) & 40000 \\
\hline & $v 12$ & 0.46 \\
\hline & $v 13$ & 0.22 \\
\hline \multirow{5}{*}{$\begin{array}{c}\text { Slip } \\
\text { constants }\end{array}$} & $\rho_{\mathrm{m}} \quad \mu \mathrm{m}^{-2}$ & 5.0 \\
\hline & $v \quad \mathrm{~Hz}$ & $1.0 \times 10^{11}$ \\
\hline & $b_{\text {basal,prism }} \quad \mu \mathrm{m}$ & $2.95 \times 10^{-4}$ \\
\hline & $b_{p y r} \quad \mu \mathrm{m}$ & $4.68 \times 10^{-4}$ \\
\hline & $k \quad \mathrm{~J} \mathrm{~K}^{-1}$ & $1.381 \times 10^{-23}$ \\
\hline \multirow{4}{*}{$\begin{array}{c}\text { Slip } \\
\text { variables }\end{array}$} & $\tau_{\text {__basal,prism }} \quad \mathrm{MPa}$ & 280 \\
\hline & $\tau_{\text {c_pyr }} \quad \mathrm{MPa}$ & 840 \\
\hline & $\Delta F \quad \mathrm{eV}$ & 0.6187 \\
\hline & $\Delta V \quad / \mathrm{b}^{3}$ & 18.75 \\
\hline
\end{tabular}

${ }^{1}$ These slip properties are based on the studies by Zhang et al. (2015c). 


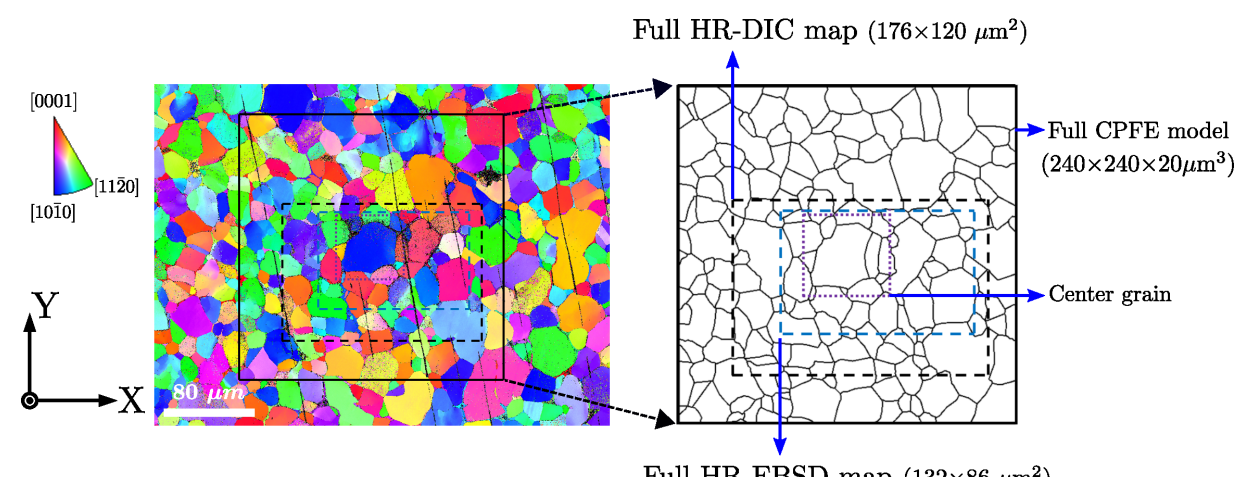

(a)

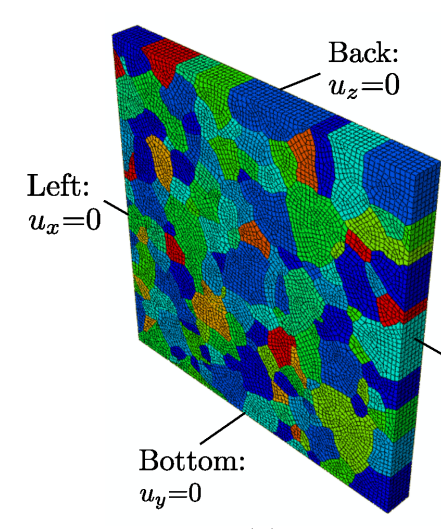

(c) (b)

Full HR-EBSD map $\left(132 \times 86 \mu \mathrm{m}^{2}\right)$

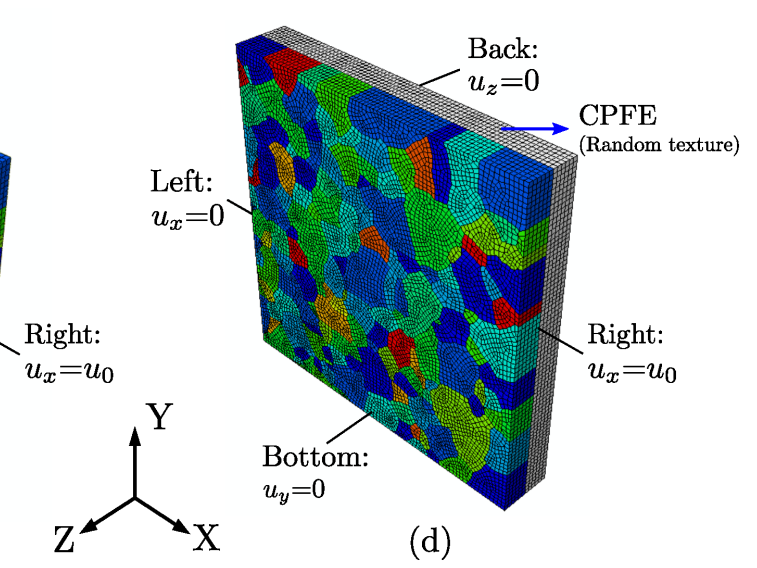

Figure 2 Tensile testing in the $\mathrm{x}$ direction and the crystal models considered: (a) inverse pole figure, along the loading direction $\mathrm{xx}$, with highlighted areas for characterisation and crystal plasticity models; (b) within the full modelled area of $240 \times 240 \mu \mathrm{m}^{2}$, HR-DIC measurement is taken in the black dashed line area, the HR-EBSD measurement within the blue dashed line area and the centre grain $\mathrm{g} 1$ demarcated by the purple dashed line; (c) the single-layer CPFE model; (d) the double-layer CPFE model with a random textured layer beneath the surface.

As shown in Figure 3(a) and Figure 3(b), the overall crystallographic texture in the tested polycrystalline material is very weak. It provides a small preference towards prismatic slip system activation, since the uniaxial loading direction is along the $\mathrm{X}$ direction in the pole figure and the c-axis of the hep crystal has a slight preferential orientation along the $\mathrm{Y}$ direction. In the experiment, the load-displacement relation is directly recorded from the microtester, using load readings taken from the $5 \mathrm{kN}$ load cell and displacements measured using a Linear Variable Differential Transformer (LVDT). The loads and displacements were transformed to stresses and strains, respectively, using the previously stated sample 
geometries. With a measured specimen gauge length of $22.67 \mathrm{~mm}$, the total average strain was calculated to be $3.3 \%$, which was applied with a strain rate $6.4 \times 10^{-5} \mathrm{~s}^{-1}$. The experimental average stress-strain behaviour is shown in Figure 4(a).

(0001)

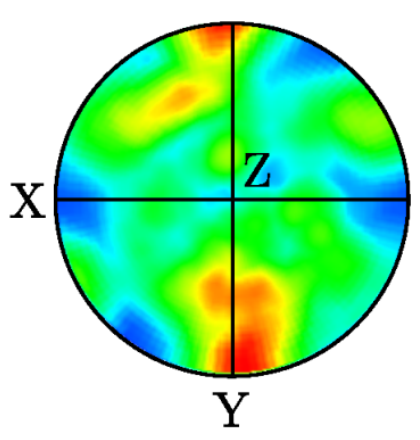

(a)
$(10 \overline{1} 0)$

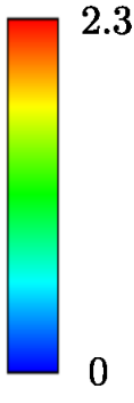

0

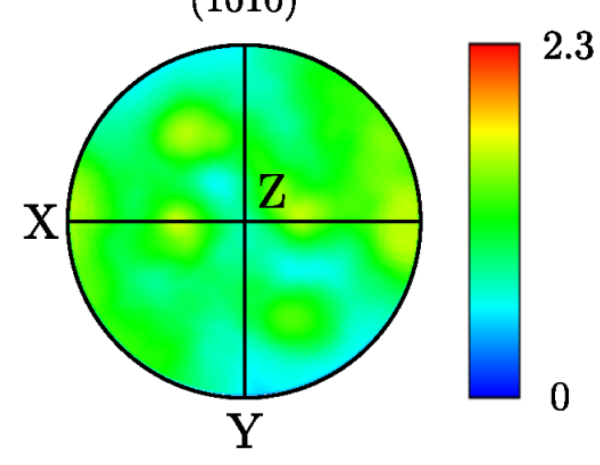

(b)

Figure 3 Texture in the tested Ti-6Al-4V polycrystalline material shown in Figure 2(a). The specimen is deformed in tension in the $\mathrm{x}$ direction. The pole figure in (a) shows majority (but not all) basal poles in plane; and in (b) the prismatic plane normal direction uniformly distributed over orientation space

In the crystal plasticity modelling, the loading was imposed in the X-direction as a linearly increasing strain-controlled tension. The total strain was taken as 3.3\% and imposed under the same strain rate as the experiment. The material properties for Ti-6Al-4V were taken from Zhang et al. (2015c), which were determined from comprehensive single crystal and polycrystal tests under differing strain rates. It is interesting to find, Figure 4, that the numerical prediction with known Ti-6Al material properties (Zhang et al., 2015c), provided in the Table 1, gives reasonable comparison with the experiment both in load-displacement and stress-strain relations and are shown in Figure 4 and (b). This is anticipated as the tested Ti-6Al-4V in this study contains $93 \%$ HCP $\alpha$ phase and thus its behaviour is expected be similar. According to the recent study on multi-phase titanium alloys (Zhang and Dunne, 2017), the macroscopic response of $\alpha+\beta$ titanium alloys (with BCC $\beta$ volume fraction less than $20 \%$ ) is dominated by the HCP $\alpha$ phase. In the present Ti-6Al-4V alloy, the $\beta$ phase 
fraction is even lower and is confined to triple points between alpha grains. Figure 4 shows the simulated response using the critical resolved shear stresses obtained independently from Gong and Wilkinson (2011) and (Zhang et al., 2015c) resulting in a higher yield point.
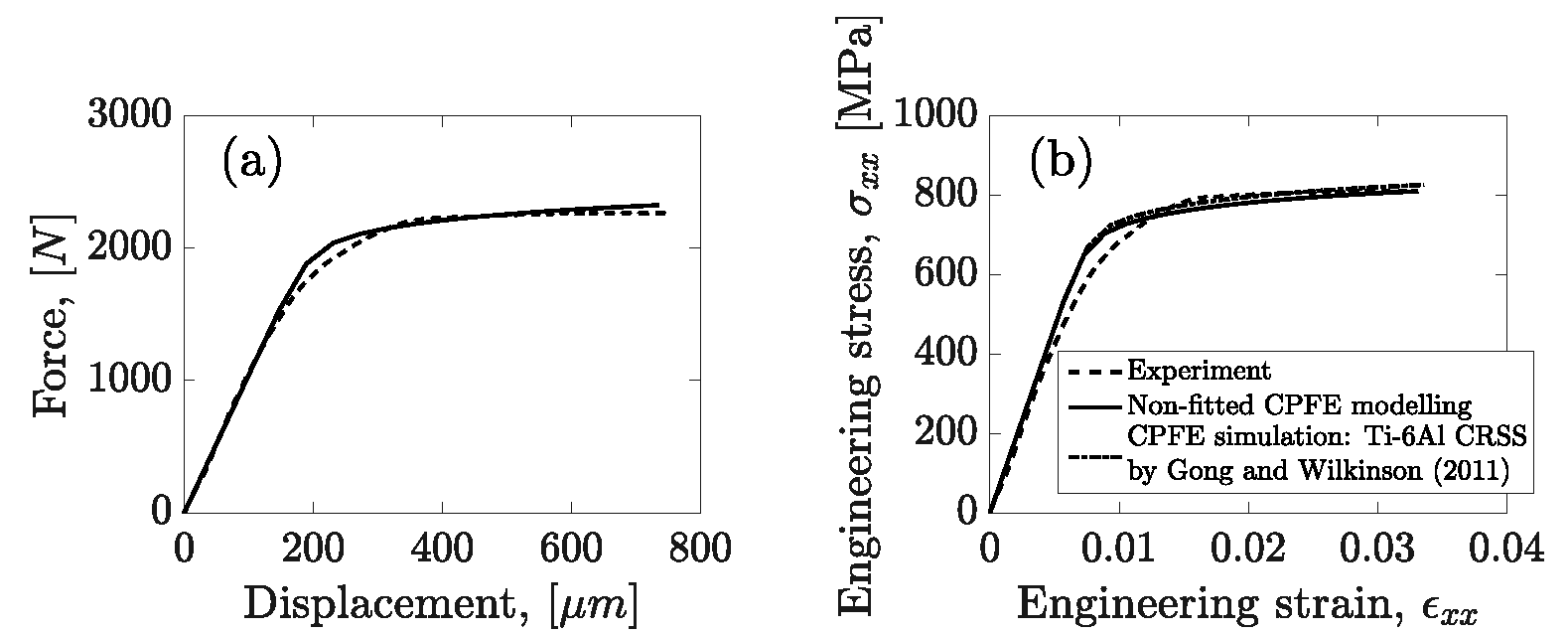

Figure 4 Numerical prediction response using non-fitted Ti-6Al material properties from Zhang et al. (2015c), and its comparison to the experiment: (a) the force-displacement relation and (b) the stress-strain curve. The total strain is obtained from averaging the strain field measured by HR-DIC. Simulation results based on Ti-6Al critical resolved shear stress (CRSS) values from Gong and Wilkinson (2011) are also shown

\subsection{Experimental and numerical slip trace analysis}

In this section, the local slip activation and slip trace analysis at the grain level are considered in order to assess the relationship between the micro-scale slip accumulation and the macroscale plastic deformation. The slip trace analysis is conducted based on the single layer crystal plasticity modelling, illustrated in Figure 2, where the slip trace at the free surface is determined based on the updated plane normals of the activated slip systems and their intersections with the free surface (Zhang et al., 2016a). The active slip systems from the HR-DIC data were determined by cross correlating slip angle projection calculated from EBSD orientation information with the slip trace angles measured from HR-DIC. The model results and the HR-DIC measurements are as shown in Figure 5. The slip traces predicted by the crystal plasticity modelling, given by the red lines in the inserted white boxes, only 
consider the slip traces provided by the primary slip activation. Note that only 6 out of 51 grains contain secondary slip activation, as observed from DIC measurements. The effect of secondary slip activation is discussed in Section 4.4 for a more detailed grain-level microscale analysis. The agreement in slip trace orientation, due to the primary slip activation, is encouraging such that about $84 \%$ slip traces out of 51 grains show good agreement between the HR-DIC measurements and model predictions. About $7 \%$ of slip traces show a deviation of $\sim 15^{\circ}$, and the remaining $9 \%$ are wrongly indicated by the model. The latter could easily be attributed to the grains below the free surface which are not explicitly represented in the modelling. However, systematic investigations of this effect are reported later. In addition, a summary analysis of experimental 'apparent' versus CP predicted Schmid factors is also presented later. Full EBSD mapping was not available for the undeformed sample, but instead was carried out after deformation. The differences are likely to be marginal considering the small deformation of $3.3 \%$ in this test.

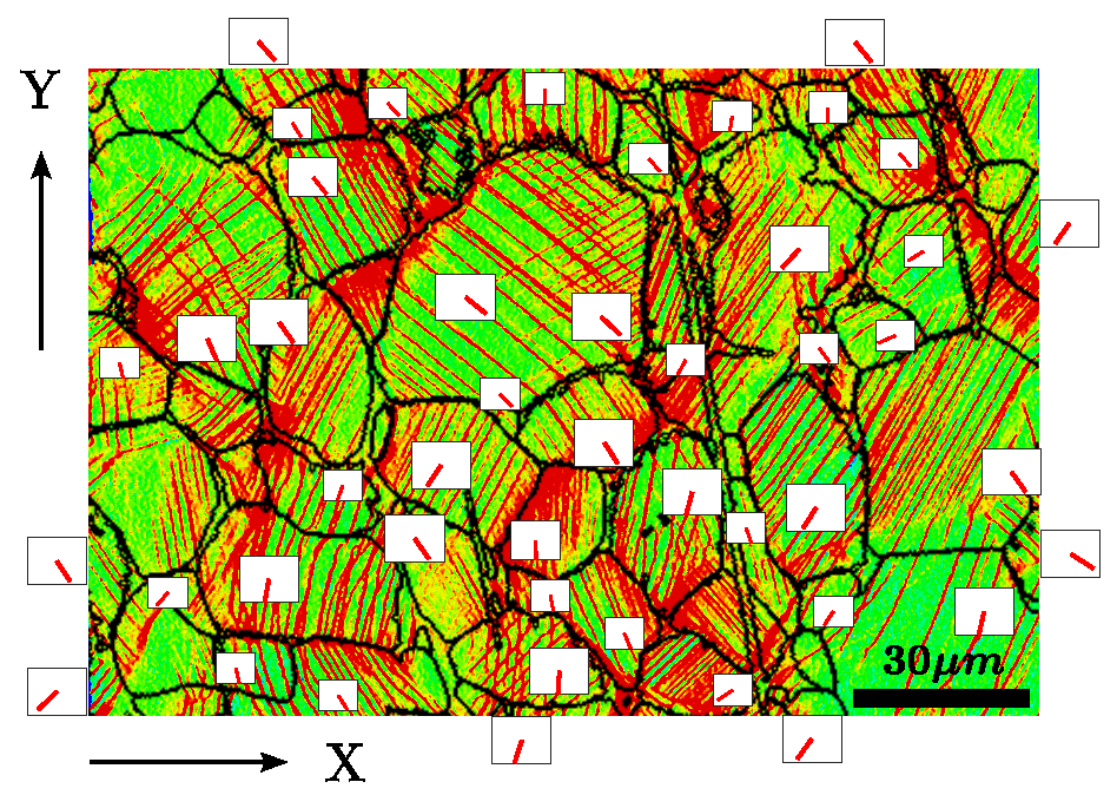

Figure 5 Slip trace (predominant) comparison by HR-DIC measurement (the red lines in the green background) and the numerical simulations (the red line in the inserted white boxes). The uniaxial tensile test is in the $\mathrm{x}$ direction. 
In order to show more quantitative results from the HR-DIC and crystal plasticity modelling, a statistical comparison of measured and calculated Schmid factor is demonstrated in Figure 6. Note that the Schmid factors obtained from the experiments are 'apparent' in that they are determined assuming a uniaxial stress state exists at the grain level. However, the $\mathrm{CP}$ results provide the full detail of (predicted) stresses and stress states, and their intergranular variations, so that Schmid factors may in principle be determined correctly at all points within each grain. However, in order to provide comparison with experiment, grainaveraged stresses are extracted from the model to determine Schmid factors. The slip activity according to HR-DIC analysis shows $35 \%$ basal, $59 \%$ prismatic and $6 \%$ pyramidal slip. The slip activation in crystal plasticity modelling is correspondingly $43 \%$ basal, $57 \%$ prismatic system and zero pyramidal system activity. Apart from the three unidentified pyramidal slip systems in the simulation, we considered all 43 grains having good agreement in primary slip activation in both experiment and simulation. As aforementioned, this corresponds to $84 \%$ of the 51 grains specifically examined. The agreement between the two is therefore reasonably close, recognising that the slip activations from HR-DIC and crystal plasticity are calculated in differing ways. The former is based on matching the slip lines from the image after deformation with those calculations from differing slip systems, with loading direction directly adopting the remote tension in the $\mathrm{X}$ direction (thereby giving approximate, apparent Schmid factors). The latter is calculated from the most active slip system such that the accumulated slip in that system is the highest, and the loading direction is affected by the local stress state. It is clear that the slip activation from the crystal plasticity modelling rightly depends on the microscale stress distribution, because the local stress state may be important in grain-level slip activation, and is often different to that assumed from the macroscale (Wan et al., 2016; Zhang et al., 2016a). 

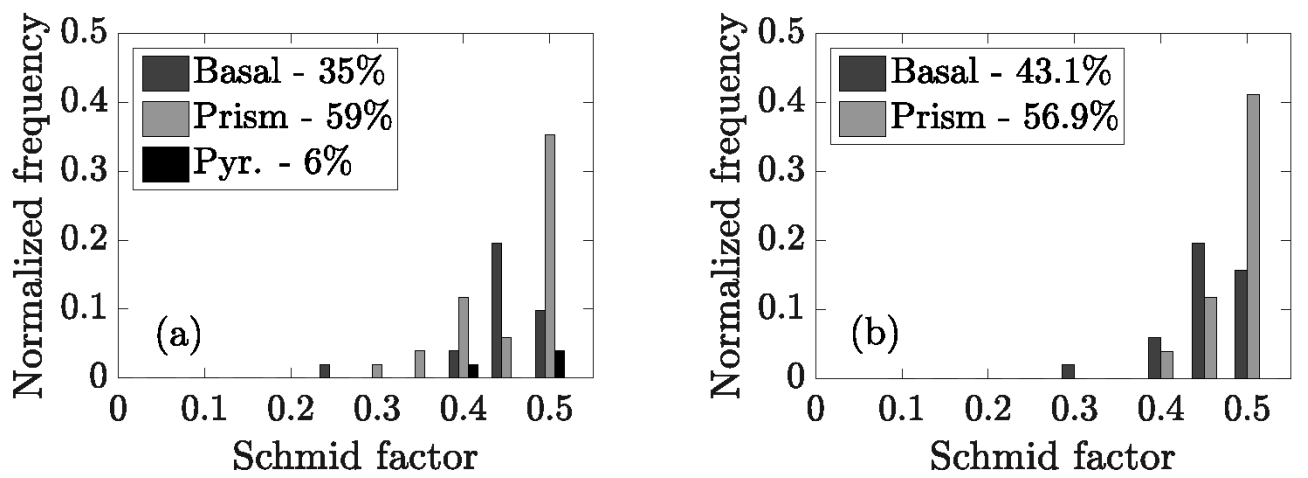

Figure 6 Histogram of slip activation in (a) experiment through HR-DIC analysis and (b) numerical modelling

Interestingly, both the HR-DIC and crystal plasticity model analyses show that the macroscopic deformation is dominated by slip systems having Schmid factors over 0.4. This agrees well with $\mathrm{Li}$ et al. (2015) in tension tests of Ti-6Al-4V at $20^{\circ} \mathrm{C}$. Greater prismatic slip system activation compared to basal slip (both have quite close CRSS values) is reasonable, since it has been indicated in Figure 3(a) that prismatic slip is favoured when tension is applied in the $\mathrm{X}$ direction. The overall slip activation is found to depend on the texture of the tested sample. For example, in their samples, Li et al. (2015) found about 47\%, 36\%, 14\% and $4 \%$ in prismatic, basal, pyramidal and twin system activation, respectively. A quantitative map of the xx-strains measured over the region of interest using the HR-DIC technique, after the applied straining, is shown in Figure 7. 


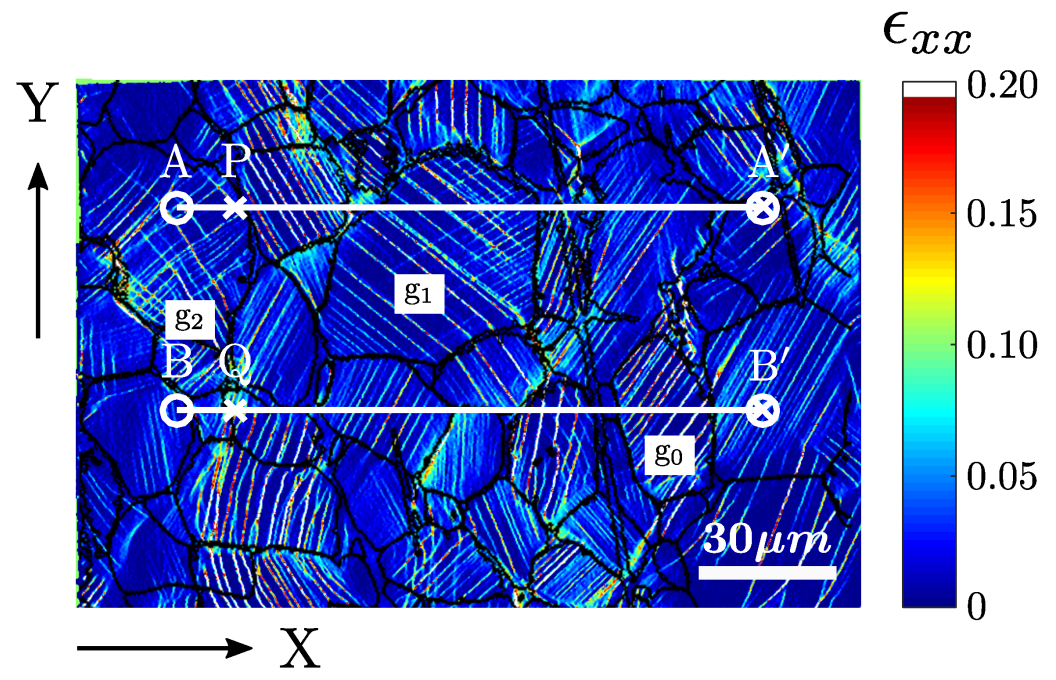

Figure 7 Slip localization from HR-DIC measurement, indicating paths A-A' and B-B' (for HR-DIC analysis) and paths P-A' and Q-B' (for HR-EBSD analysis). Crystal plasticity modelling data is available for both sets of paths (below). The uniaxial tensile test is in the $\mathrm{x}$ direction.
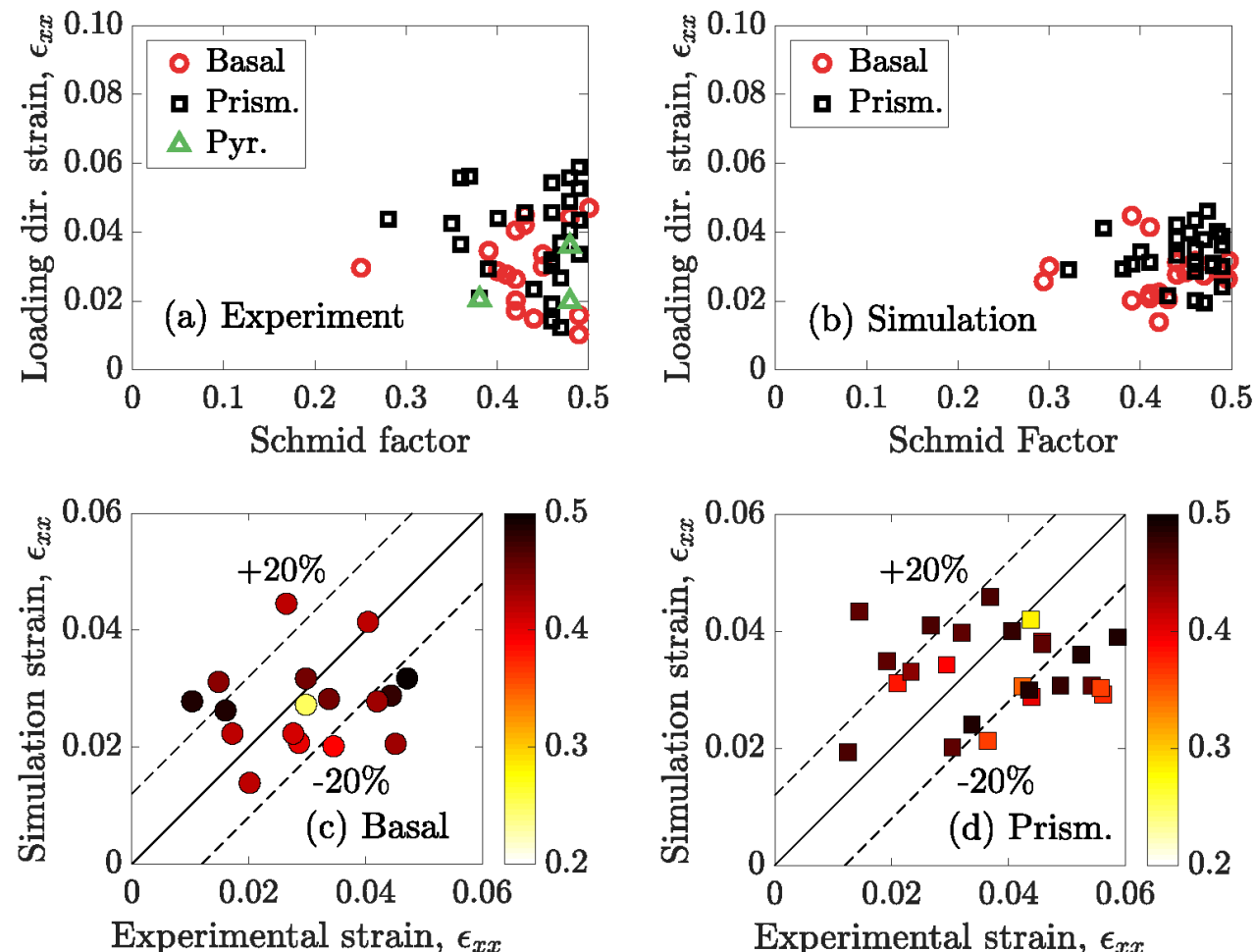

Figure 8 shows (a) experimental HR-DIC and (b) CP model grain-averaged loading direction strains resulting from the Schmid factor magnitudes shown ('apparent' for the experimental results i.e. based on a uniaxial stress state, and grain-averaged for the model) and the slip system type; and (c) basal and (d) prism slip activations contributing to the $\mathrm{xx}$-strains obtained in experiment and modelling, for Schmid factors shown by the colour scale. 
Figure 8 shows the relationship between grain-averaged strain, obtained from Figure 7, and Schmid factor magnitude for the HR-DIC and CP modelling in (a) and (b) respectively. In the analysis of the HR-DIC results, the $\sim 150 \mathrm{~nm}$ interrogation distance determines the length scale over which displacements (and hence strains) are measured. Grain-averaged DIC strains are calculated by summing all such strains of a given component, over the grain area, divided by the number of strain measurements in the region. In the CP modelling, strains are averaged over all elements within the relevant grain. Again, the same 43 out of 51 specifically examined grains are considered as in Figure 6. It is clear from both the experimental observations and CP modelling that higher Schmid factors (be that apparent, based on the uniaxial stress assumption in the experiments, or grain averaged and determined from maximum principal stress in the $\mathrm{CP}$ calculations) result in the majority of the (grainaveraged) straining. In addition, prism slip is found to lead to higher strains generally than that from basal activation, and this is apparent from both DIC measurement and CP modelling, showing reasonably good agreement. Figure 8 (c) and (d) show the comparisons for the grain-averaged strains determined from the HR-DIC measurements and the CP modelling, such that some agreement within about $20 \%$ is achieved for all the grains considered. The strains developed within both predominantly basal and prism slip grains in these figures again generally show that the higher magnitudes typically arise from the higher Schmid factors for both basal and prismatic slip. However, prismatic slip seems to contribute more to the higher grain-averaged loading direction strains.

As shown in Figure 6(b) and Figure 8(b), pyramidal systems are not activated according to the crystal plasticity model, noting that the critical resolved shear stress for this system is about three times higher than that for the basal and prismatic systems (Zhang et al., 2015c), which has also been previously reported in micro-cantilever experiments on titanium (Gong 
and Wilkinson, 2009). Three grains have been identified from the HR-DIC analysis for which pyramidal slip has been observed, for which the apparent Schmid factors are determined to be $0.48,0.48$, and 0.38 , respectively. The corresponding average xx-strains in these grains are found to be $0.036,0.02$, and 0.021 , respectively. The local average Schmid factors from CP modelling for the pyramidal systems in the corresponding grains are found to be $0.27,0.47$, and 0.46 , respectively. Thus, two of the three grain orientations result in high pyramidal slip Schmid factors, but the resolved shear stresses are simply not high enough in the CP modelling to exceed the critical resolved shear stresses in order to activate slip. The average strains obtained from the CP modelling, but therefore derived from basal and prism slip (since pyramidal slip is not activated), are $0.026,0.029$, and 0.03 , respectively.
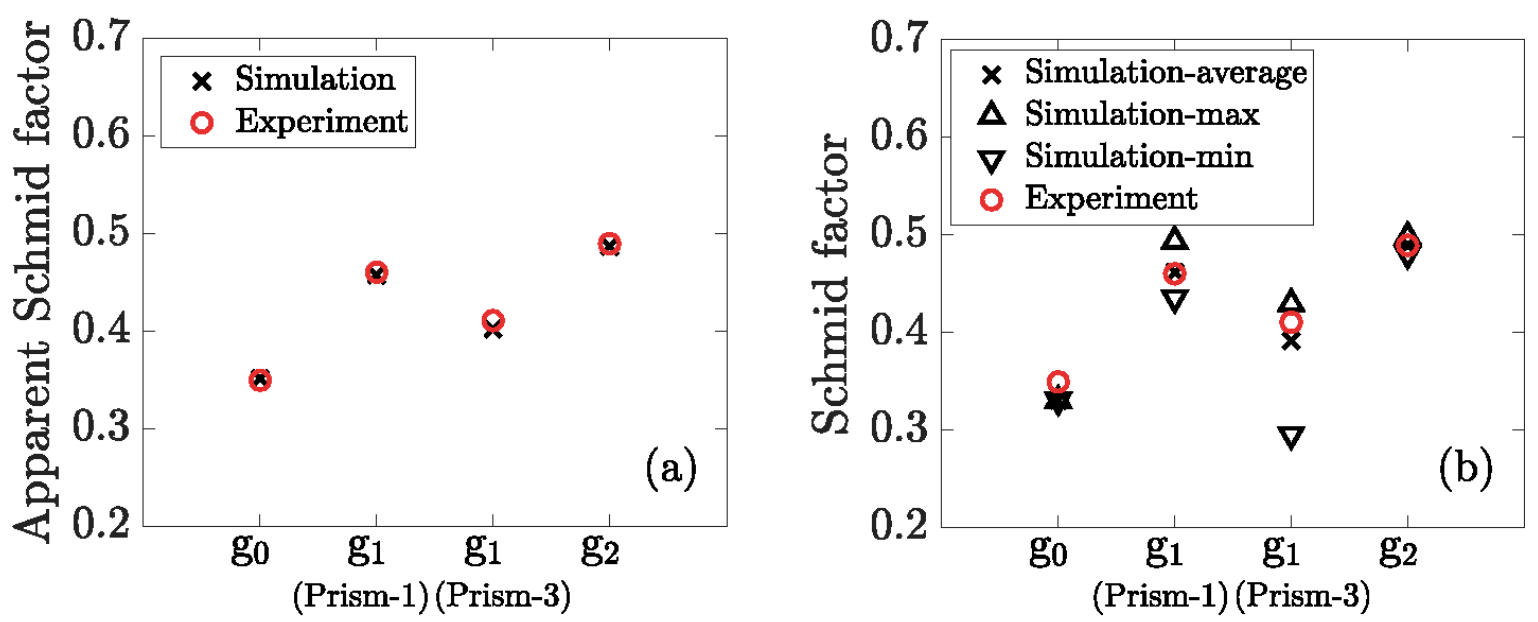

Figure 9 The Schmid factors in three grains labelled in Figure 7 are compared between CP modelling and the HR-DIC experiment for the activated primary slip system: (a) the apparent Schmid factors, both calculated using remote loading direction and (b) local Schmid factor by modelling shows variation in values for all three concerned grains (their locations are shown in Figure 7) compared to the apparent Schmid factor obtained from experiment. "Prism-1" and "Prism-3" are two activated prismatic slip systems in grain g1 (corresponding to Figure 14(f) and (g)), respectively.

A quantitative comparison of Schmid factors obtained from CP simulation and experiments is shown in Figure 9 for grains labelled in Figure 7. Experimentally determined 
Schmid factors come from the assumption that the stress state remains uniaxial (reflecting the applied loading) whereas the CP modelling takes full account of the local, microstructurallevel stress state (which is often not uniaxial). In the latter case, therefore, the Schmid factors are determined utilising the resultant maximum principal stress direction determined locally within the grain of interest. Interestingly, as shown in Figure 9, the CP model predicted range of Schmid factor for grains g0 and g2 is low, reflecting reasonably uniform stress state in these grains. As a consequence, the Schmid factors calculated show close agreement with the experimental 'apparent' Schmid factors. However, for grains g1 (prism-1) and g1(prism-3), the stress inhomogeneity within this grain is larger such that the CP predicted range of Schmid factors is larger, reflecting the fact that the stress state is not uniaxial in these grains. But the average grain Schmid factor is in fact close to the experimental 'apparent' Schmid factors, indicating that the average stress state across these grains remains approximately uniaxial. However, it is also clear that intragranular analysis of Schmid factor, and hence slip activation, generally requires knowledge of the full stress state and that e.g. in areas of high elastic constraint, the apparent Schmid factors can be quite different, as shown in Figure 9.

\subsection{Grain level strain, lattice rotation and GND analysis}

This section provides a quantitative comparison of the material response from a region common to both the HR-EBSD and HR-DIC measurements. Two paths are selected for further analysis and are highlighted by solid white lines in Figure 7. Paths A-A' and B-B' contain HR-DIC measurement and Paths P-A' and Q-B' provide both HR-DIC and HREBSD data. Figure 7 shows the strain map in the loading direction obtained from HR-DIC measurements. Apart from the clear observation of high-strain slip traces within the majority of grains, there are also significant concentrations of high-strain accumulation near grain boundaries, and in particular, at triple junctions. 


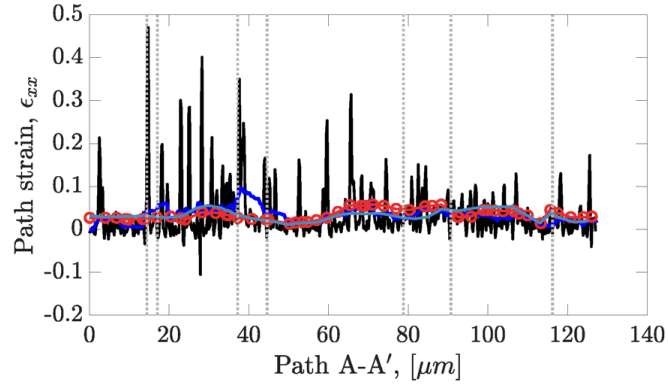

(a) $\epsilon_{x x}$ : top path A-A'

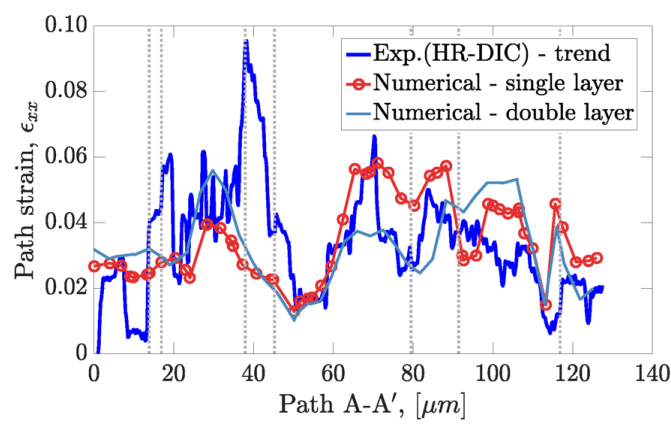

(c) $\epsilon_{x x}:$ top path $\mathrm{A}-\mathrm{A}^{\prime}((\mathrm{a})$ enlarged)

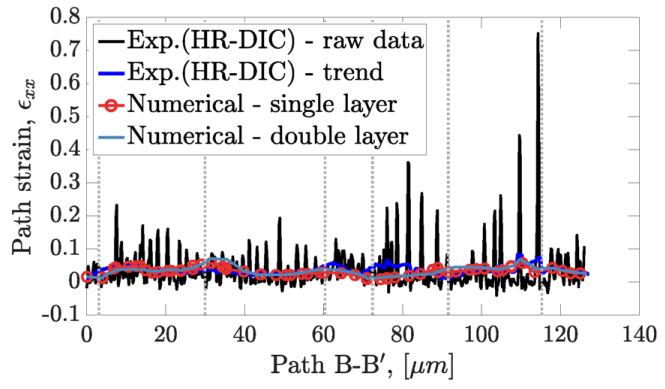

(b) $\epsilon_{x x}$ : bottom path B-B'

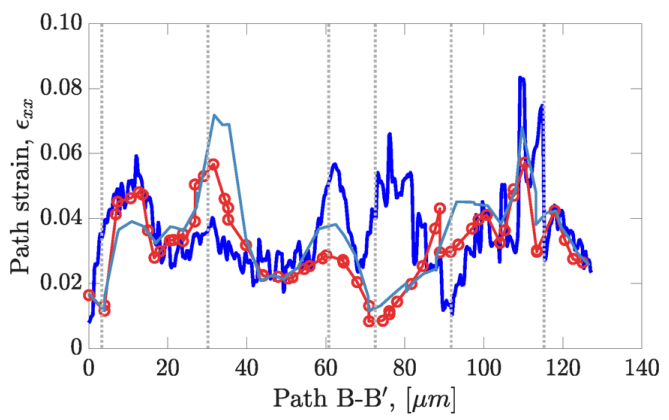

(d) $\epsilon_{x x}$ : top path B-B' ((b) enlarged)

Figure 10 Microscale xx-strains from HR-DIC and CP numerical modelling (from the free surface layer of elements) along paths (a) A-A' and (b) B-B' shown in Figure 7. (c) and (d) show the DIC trend and model results but without the DIC raw experimental data. Single layer indicates just one layer of grains included in the modelling; double layer indicates a second layer of grains assigned random crystallographic orientation illustrated in Figure 2(d). The vertical grey dash lines indicate grain boundaries.

Since the resolution in HR-DIC is much higher than that in the numerical simulation, capturing nanoscale detail and the discrete nature of the slip system activation (with highstrain peaks from individual slip traces clearly observed in Figure 10(a)), it is necessary to process the experimental data from HR-DIC to provide a like-for-like comparison. For this reason, the raw HR-DIC data is coarsened by averaging over 30 data points in a given neighbourhood. This gives a corresponding path length of about $4 \mu \mathrm{m}$ over which the DIC smoothing is carried out, and corresponds reasonably to the size of a single finite element in the model. To investigate the role of grains beneath the free surface, in the analyses presented in this section, the sub-layer of grains described earlier (Figure 2(d) in Section 4.1) with randomly assigned crystallographic orientations is both included and excluded in the 
modelling in order to assess the sub-layer effect on free-surface calculated response. As shown in Figure 10(a) and (b), the xx strains along the two paths shown, taken from the surface layer of elements in modelling, agree reasonably well with the HR-DIC measurement, except that the detailed slip distributions around triple junctions do not appear to be well represented. Note that the observed local stress and strain fields at the free surface are affected by the orientation of the underlying grains in the volume (St-Pierre et al., 2008; Zeghadi et al., 2007). Model results are always taken from the free surface in this study in order to provide comparison with the experiment. It is also seen that the inclusion of a sublayer of grains with a random texture does have an effect on the CP-calculated strain observed on the free surface. This is seen more clearly in Figure 10(c) and (d). The effects of the crystallographic orientation may be important for other quantitative measures such as elastic strain, stress, lattice rotation and GND density, and these effects are considered further below. In addition, the misorientations which develop within individual grains can be as high as $15^{\circ}$, and are likely also to be important in influencing local quantities; this is discussed in Section 4.4.

A study of lattice rotations, curvatures and GND densities has been conducted utilising both crystal plasticity modelling and HR-EBSD measurements at the grain-level. Figure 11(a) and (b) show that the relative lattice rotation (with respect to the mean lattice rotation) from HR-EBSD is reasonably well represented along paths A-A' and B-B' by the CP modelling for both the single free-surface layer of grains and the model including the additional sublayer of random grains. The inclusion of the sub-layer crystallographic heterogeneity affects the magnitude of relative lattice rotations, but it does not appear significantly to change the trend along paths A-A' and B-B'. The crystal plasticity model calculated GND density and HR-EBSD measured results are shown in Figure 12. In a manner similar to that used for processing the HR-DIC trend lines, the trend line of GND density from HR-EBSD is 
obtained by averaging over 10 data points in a given neighbourhood. This gives a corresponding path length of about $4 \mu \mathrm{m}$.

The GND densities are typically found to be higher at grain boundaries when compared to the interior of grains, reflecting strain localisation and the development of plastic strain gradients at grain boundaries. The differences in Figure 12 may reflect the absence of information on the initial sample heat treatment giving rise to a distribution of GND density in the undeformed state prior to the applied loading imposed for the modelling. In addition, we note that the HR-EBSD method gives six components of the Nye tensor whereas CPFE model gives all nine. Similar to the case for the relative lattice rotation, the inclusion of the sub-layer of randomly orientated grains below the free surface has little impact on the resulting surface GND density distributions according to the $\mathrm{CP}$ modelling. Many studies (Miller and Dawson, 2014; Yang et al., 2011; Zhang et al., 2015a) have argued that it is important to reflect both crystallography and morphology in sub-layer grain modelling so that it is somewhat surprising to find the limited impact of the grain sub-layer on the surface GND distributions.
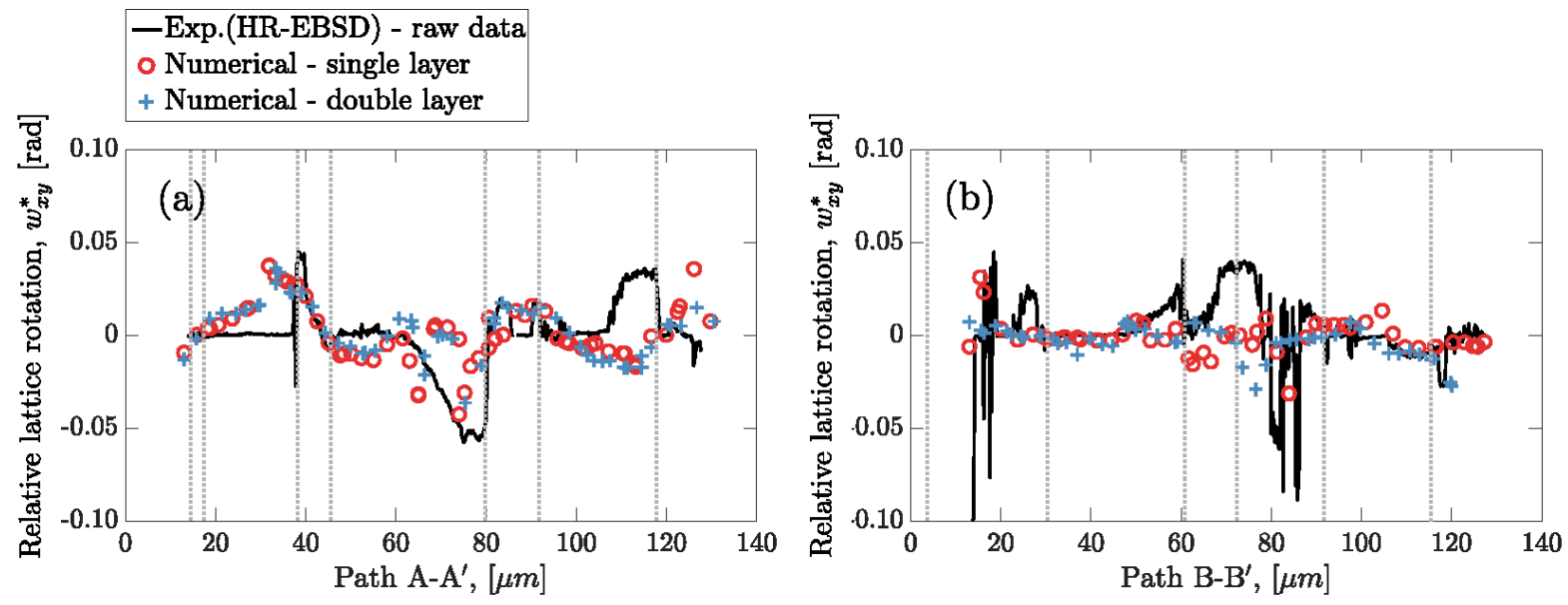

Figure 11 The relative lattice rotation along paths (a) A-A' and (b) B-B'. Note the relative lattice rotation is given by the difference between pointwise rotation and the grain average lattice rotation. Single layer indicates just one layer of grains included in the modelling; double layer indicates a second layer of grains assigned random crystallographic orientation illustrated in Figure 2(d). The vertical grey dashed lines indicate grain boundaries. 

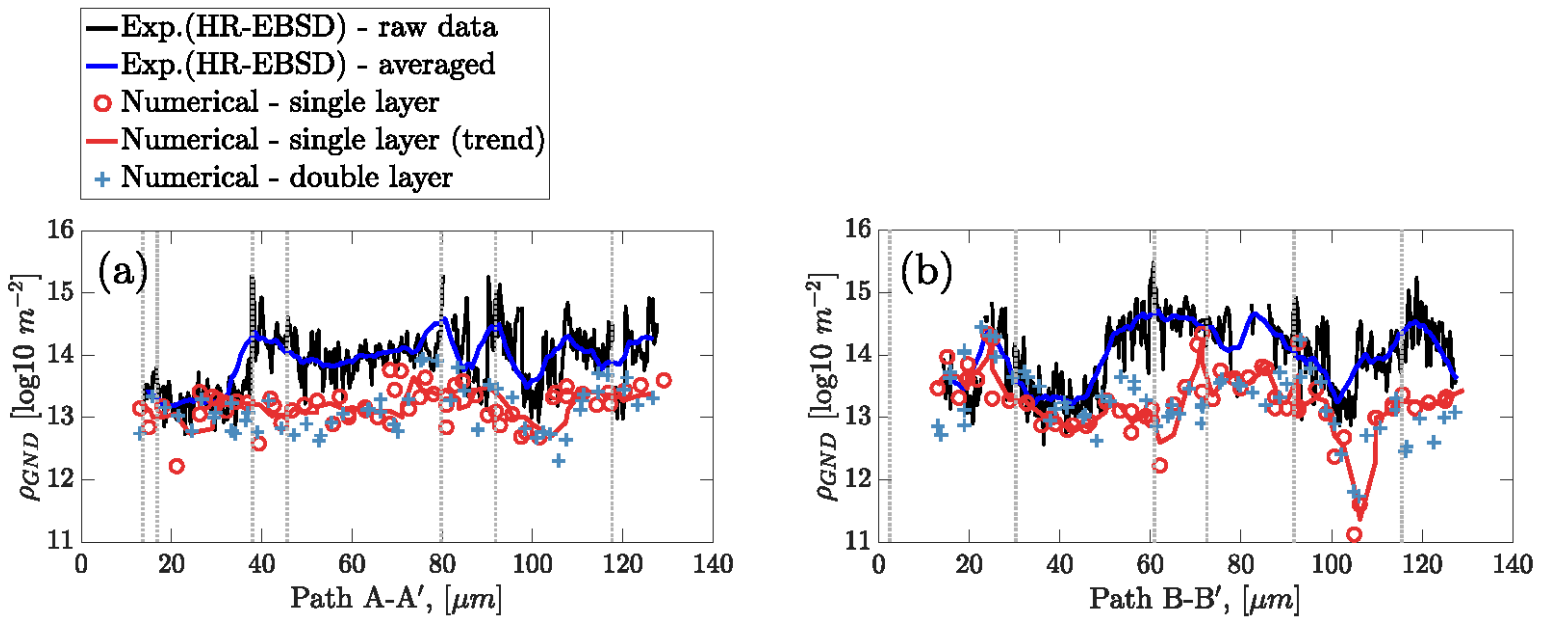

Figure 12 The GND density along paths (a) A-A'and (b) B-B'. Single layer indicates just one layer of grains included in the modelling; double layer indicates a second layer of grains assigned random crystallographic orientation illustrated in Figure 2(d). The vertical grey dashed lines correspond to grain boundaries.
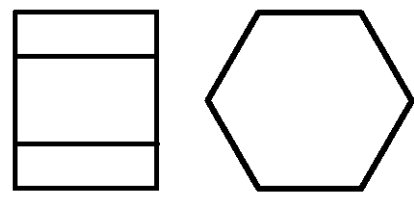

(a) hard

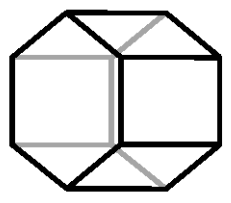

(c) basal
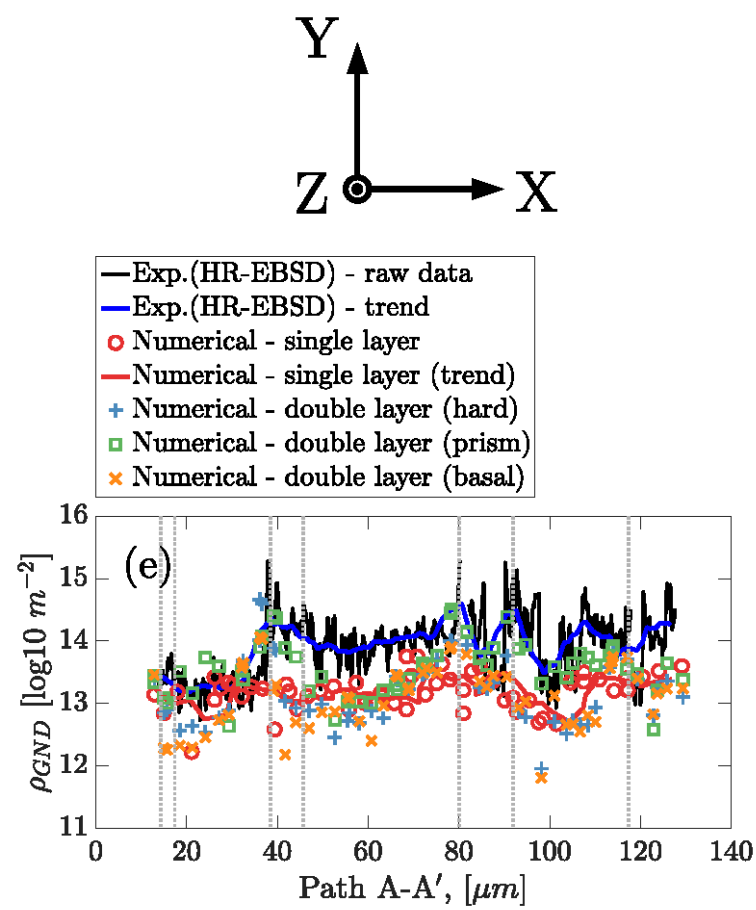
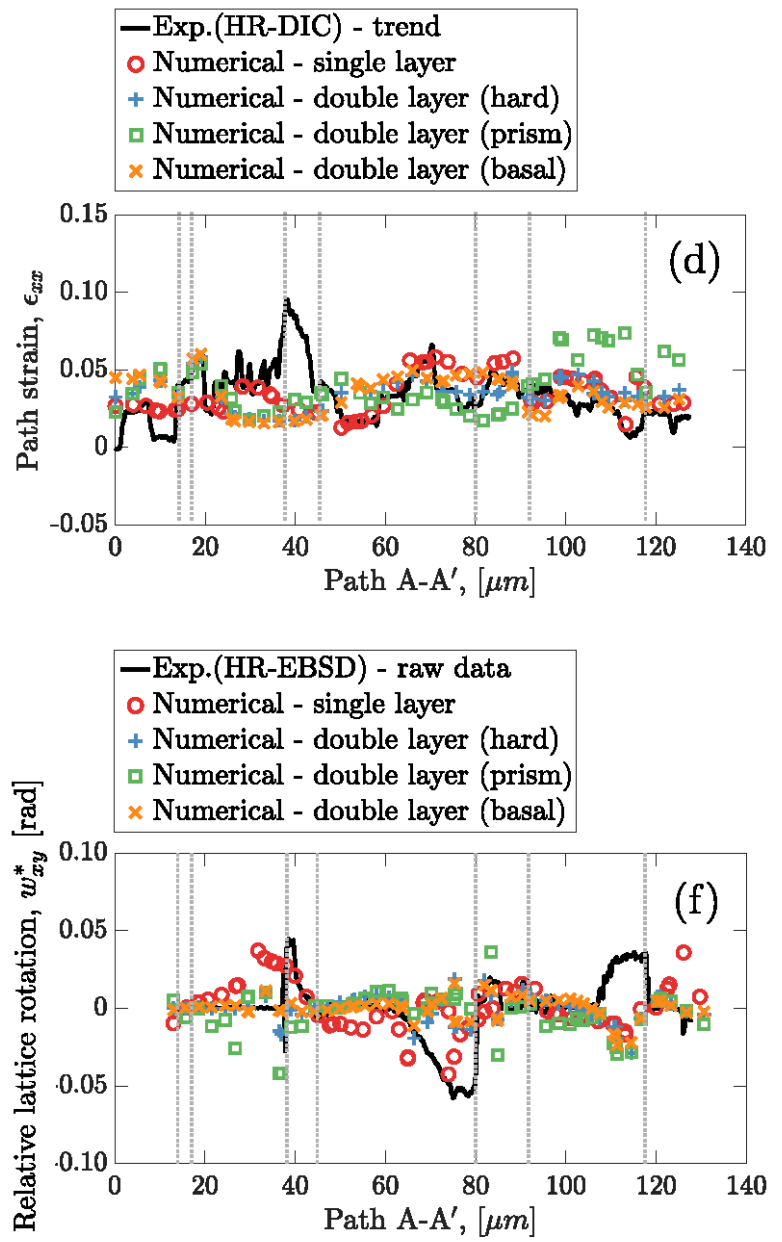
Figure 13 Surface measurement along A-A' from HR-EBSD and HR-DIC, and CP modelling based on differing model grain sub-layer extreme (single crystal) textures indicated in (a) hard orientation (c-axis parallel to loading), (b) soft orientation (c-axis normal to loading), and (c) a second soft orientation, respectively. The surface measurements along A-A' are: (d) xx-strain; (e) GND density and (f) relative lattice rotation, respectively. The vertical grey dashed lines indicate grain boundaries.

It is seen from Figures 10 to 12 that the surface strains, GND densities and lattice rotations are locally affected by the presence of the sub-layer of randomly oriented grains. As a consequence, further $\mathrm{CP}$ modelling was carried out to examine the effect of sub-layer textures on free-surface results in order to examine the bounds on behaviour. As shown in Figure 13(a), (b) and (c), the grain sub-layers were chosen to represent extremes of texture by considering single crystal orientation for the sub-layer with crystal c-axis parallel to the loading direction (a hard grain configuration with no/limited slip in the sub-layer), c-axis normal to the loading (a soft orientation with prism slip in the sub-layer) and c-axis oblique to both loading and normal directions (giving an alternative soft orientation with basal and prism slip in the sub-layer). Considering the loading in the x-direction, the soft sub-layer orientations, illustrated in Figure 13(b) and (c), can lead to in-plane (x-y) and out of plane slip respectively. Figure 13(d) shows that the $\mathrm{x}$-direction strain response at the free surface is significantly affected by the three sub-layer orientations but that the responses are consistent but with local fluctuations. For the sub-layer which is soft in-plane, i.e. Figure 13(b), the corresponding out-of-plane deformation becomes elastic and inhibits out of plane slip. It is thus seen in Figure 13(e) that the GND densities are pushed up at grain boundaries as a result of the elastic constraint imposed in the out-of-plane direction by the hard sub-layer. Correspondingly, the in-plane lattice rotation, shown in a Figure 13(f), decreases. Clearly the crystallographic orientation/texture of the sub-layer affects the free-surface observations. But often, the influence may be confined to localised grain events as shown in Figure 10 to Figure 12 for the case of a random texture assigned to the sub-layer. The influence is more 
significant when strong textures exist in the sub-layer, introducing considerable additional constraint on the free-surface deformation as in the case in Figure 13.

4.4 Local grain-level slip localization analysis: single, multiple, and wavy slip traces

The polycrystalline-level slip trace analysis in Section 4.2 shows that reasonably good agreement is achievable between experimental observation and crystal plasticity modelling of slip activation. As shown in Figure 5, the slip activity in most grains is dominated by a single primary slip system and the corresponding slip trace is shown as a single straight line. However, the experimental slip traces in some grains have indicated the activation of multislip. It is noted that in the modelling of polycrystalline behaviour, it has previously been suggested that crystal plasticity is not capable of representing grain-level slip traces (Di Gioacchino and da Fonseca, 2015). In addition, wavy slip traces are often observed in experimental testing, for example as in Figure 5, and the role of grain level re-orientation on the formation of slip traces is also of interest. In this section, three representative grains for which single (straight) slip, multi-slip (straight), and wavy slip traces occur are selected for detailed study using the CP modelling.

Three grains in the central region of Figure 2 that are far from the imposed boundary conditions were examined in greater detail, and identified as grains g0, g1, and g2 and labelled in Figure 7. The finite element mesh in grain g1, which shows evidence of multi-slip activity, was refined such that a representative element (20-noded C3D20R element) size is 2 $\mu \mathrm{m}$. Two additional grains have also been considered which capture single slip behaviour and more complex multiple slip with curved traces respectively, as shown in grains g0 and g2 in Figure 7. For the case of single slip in grain g0, Figure 14(a) and (b) show interesting comparisons between the $\mathrm{xx}$ strains from HR-DIC and the $\mathrm{CP}$ model result. In particular, 
closely spaced slip traces develop in the highly deformed top left corner of this grain in the HR-DIC measurement, which exhibits as highly deformed red shades in the simulated results. At the bottom-right of this grain, more sparse slip traces are seen to develop from the HRDIC measurement for which the CP model predicts lower strains given by Figure 14(b). The $\mathrm{xx}$ strain field has been found to develop predominantly from prismatic slip given in Figure 14(c), for which the activated slip plane and normal in the hexagonal unit cell are also inserted. The central grain g1, which exhibits multi-slip is shown in Figure 14(d), and the two slip traces observable are caused by two differing prismatic systems illustrated in Figure 14(f) and (g). The accumulated slip fields are shown separately in Figure 14(f) and (g), though they cannot be understood unambiguously from the resulting strain field in Figure 14(e). The most complex case is demonstrated in Figure $14(\mathrm{~h})$ for grain g2 where the total deformation is accommodated by multiple curved slip traces. It is seen from Figure 14(i) showing the overall $\mathrm{CP}$ model calculated xx-strains that the curved nature of the slip trace at the top of grain $\mathrm{g} 2$ has been reasonably well reproduced in the numerical simulations. From Figure 14(j) and (1), the curved slip traces can be seen to derive from the contribution of a basal and a prismatic slip system, respectively. The combination of these two slip systems gives rise to the curved strain trace in the map in Figure 14(g), though the slip directions of the two activated slip systems are the same. Basal slip was activated first (according to the $\mathrm{CP}$ model) at the top of the grain, followed by the prismatic slip system, which was found to give rise to slip superposition. The basal and prismatic slip systems, which are activated and give rise to the wavy slip in Figure 14(h), are quantified and shown diagrammatically in Figure 15. The slip systems have been projected in to the free surface of the sample, i.e. the red lines in Figure 15, in order to show the relationship between the curved slip trace and the two originating slip systems, demonstrating the magnitude of the slip trace curvature observed. The other two accumulated slip fields in Figure 14(n) and (p), corresponding to the 
inserted basal slip and prismatic slip in Figure 14, are responsible for the localized slip at the bottom of the grain in Figure 14(h).

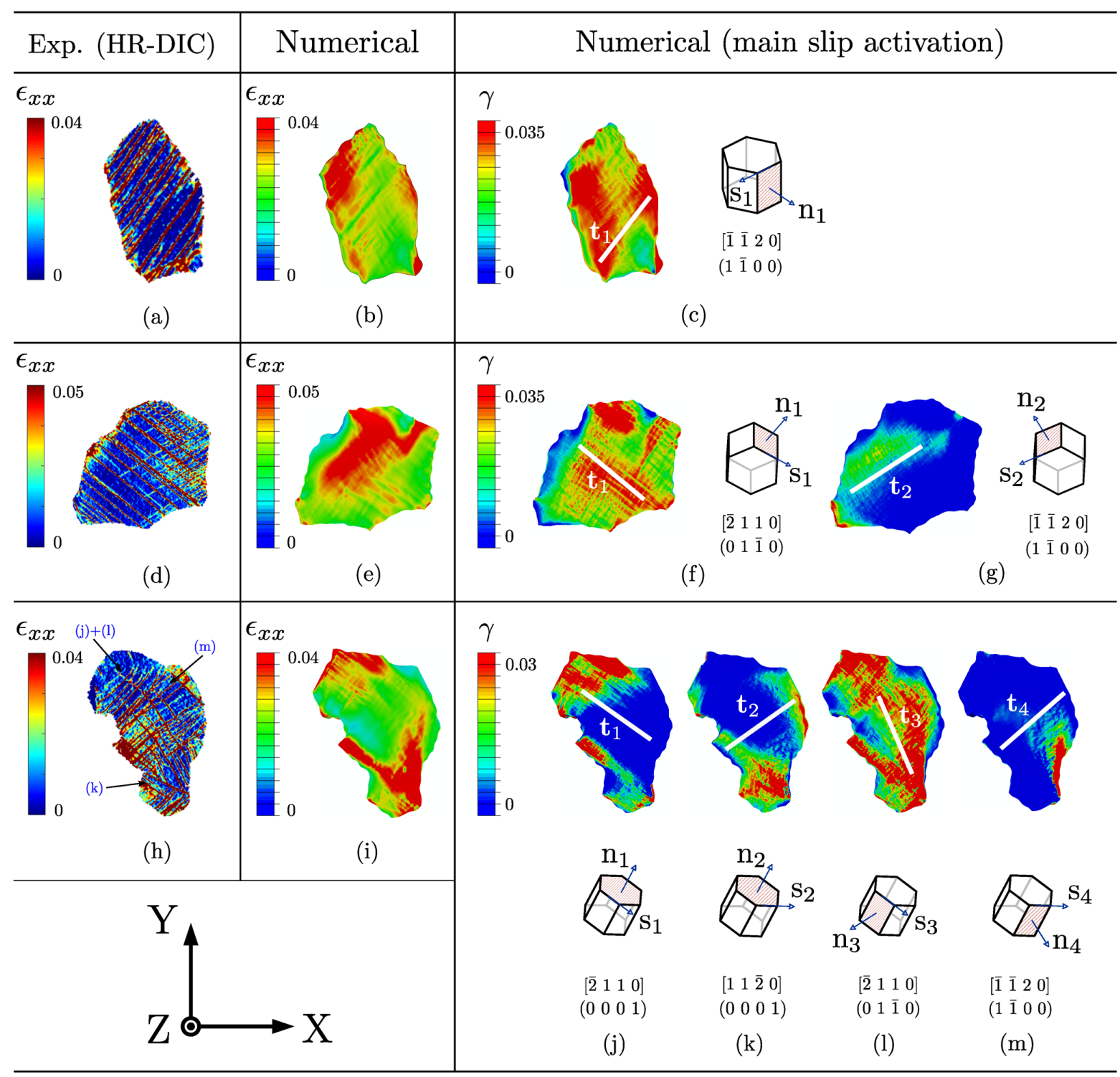

Figure 14 Crystal plasticity modelling interpretation of HR-DIC measurement for single(straight), multi- (straight), and multiple wavy slip in three representative grains g0, g1, g2 in rows in (a), (d), and (h), respectively. The numerical slip traces for the main slip activation have been highlighted in white lines. The uniaxial tensile test is in the $\mathrm{x}$ direction. 


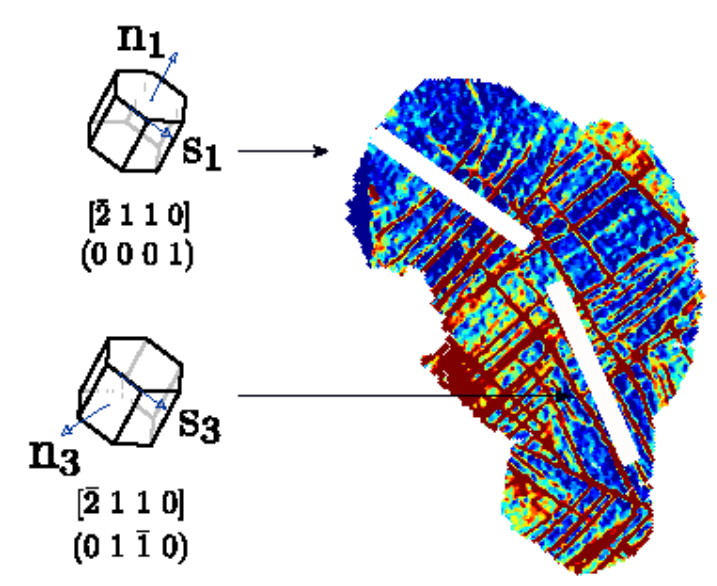

Figure 15 Slip interaction in grain g2: the wavy slip trace in Figure 14(h) is introduced by the superposition of a basal slip and a prism slip, as shown in Figure 14(j) and Figure 14 (1), respectively. The white lines herein show the projection of the slip planes of these two systems on to the free surface.

With reference to the large central grain g1, the HR-DIC trace analysis shows that the left hand side of the grain develops prismatic $<\mathrm{a}>$ slip with an apparent (i.e. assuming a local uniaxial stress state corresponding to the applied uniaxial loading) Schmid factor of 0.46, as shown in Figure 14(f), and the right hand side deforms by an alternative prismatic slip system, as shown in Figure $14(\mathrm{~g})$, with a global Schmid factor of 0.41 . These correspond well to those calculated in the CPFE modelling (which does take full account of the local stress state) which are 0.462 and 0.39 , respectively. These two systems, Figure 14(f) and (g), have all been highlighted as prism-1 and prism-3 in Figure 9 in grain g1.

Figures 11 and 13 above have shown the development of heterogeneous lattice rotation resulting from constrained slip within the polycrystal, thus giving rise to the development of misorientation and grain spatial misorientation distributions. From EBSD mapping and resultant knowledge of pointwise crystal orientation, the misorientation between two crystal points is defined as $\phi=\operatorname{acos}\left[\frac{\operatorname{tr}(\Delta \mathbf{g})-1}{2}\right\rfloor$ where $\Delta \mathbf{g}=\mathbf{g}_{0}^{-1} \mathbf{g}_{i}$ and $\mathbf{g}_{0}$ is the orientation matrix of a reference location, and $\mathbf{g}_{i}$ that corresponding to the point of interest (either within the same grain or elsewhere). In what follows, misorientations are investigated by considering the 
appropriate orientation matrices, $\mathbf{g}_{0}$ and $\mathbf{g}_{i}$. Firstly, a reference point is selected in a given grain (defining $\mathbf{g}_{0}$ ), and then a path chosen along which the deviation of the pointwise orientation $\mathbf{g}_{i}$ from that given by $\mathbf{g}_{0}$ is calculated using the above expression for misorientation, giving the path misorientation. The path misorientations determined from EBSD characterisation and orientation mapping in the three grains of interest listed in Figure 14 are considered and are shown in Figure 16(a). The path misorientation is rather low in grain g0, where single slip is indicated by the traces which have been demonstrated in Figure 14(a). The peak path misorientation along path C-C' has been found to be about $3^{\circ}$. The path misorientation in grain g1 has been found to be high at the right-hand side of the grain, as demonstrated in Figure 16(b). The maximum path misorientation is higher than $5^{\circ}$ along Path D-D' in Figure 16(e). The path misorientation in grain g2, containing complex multiple curved slip traces, shows significant heterogeneous misorientation as illustrated in Figure 16(c). It is found that the greatest path misorientation can be as high as $15^{\circ}$.

Figure 16(a) and (c) show considerable differences in grain-level misorientation, with grain g2 showing very significant misorientation, assumed to result from the imposed deformation. Unfortunately, however, full EBSD mapping was not available for the undeformed sample and each grain orientation in the undeformed state has been taken to be uniform in the $\mathrm{CP}$ modelling. To study the role of intra-grain misorientation on the measured free surface response, further $\mathrm{CP}$ models have been established for grain g2, which has the greatest misorientation. Two cases are considered for examining the misorientation distribution effect. Firstly, grain g2 is taken to have completely uniform orientation prior to imposed loading in the modelling as shown in Figure 17(a). Secondly, the EBSD-measured misorientation shown in Figure 16(c) is explicitly represented in the CP model prior to the applied loading, shown in Figure 16(c), representing an initial misorientation distribution (having 7 different sub- 
grains with slightly differing crystal orientations as shown in the figure). The resulting accumulated plastic strain fields after loading for the two cases for grain g2 are shown in Figure 17 (b) and (d), respectively. It is found that the calculated accumulated plastic strains after loading for these two grain misorientation cases are reasonably close but that some strain localization differences are observed local to grain boundaries.
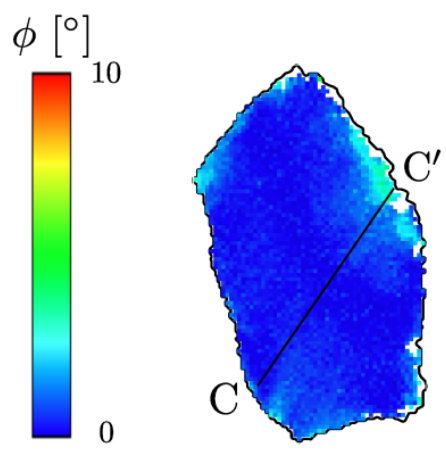

(a)

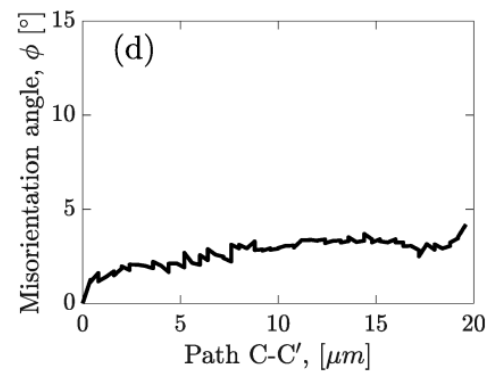

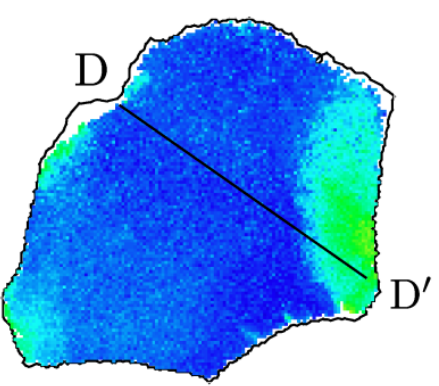

(b)

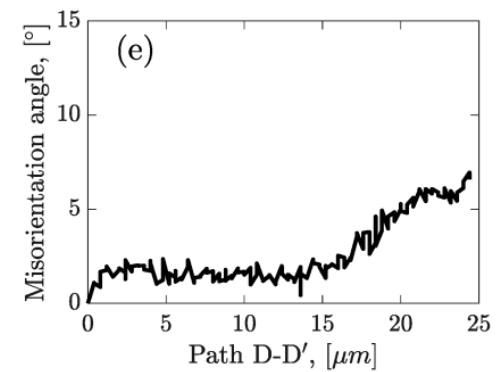

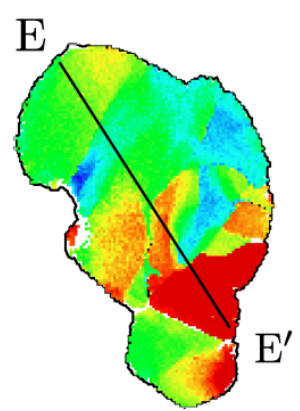

(c)

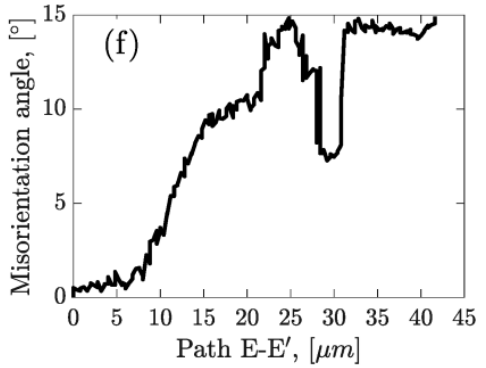

Figure 16 Misorientation in three representative grains g0, g1, and g2 in Figure 7: full misorientation map is shown in (a), (b) and (c), respectively, and the path plot on these grains on C-C', D-D', and E-E' are correspondingly extracted in (d), (e) and (f). For instance in path $\mathrm{C}-\mathrm{C}$ ', the misorientation being shown here is the angle between any pixels along $\mathrm{C}-\mathrm{C}$ ' wrt the reference $\mathrm{C}$. 


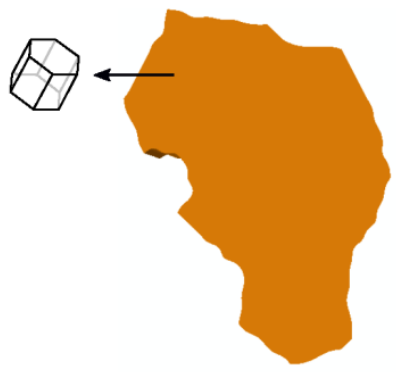

(a)

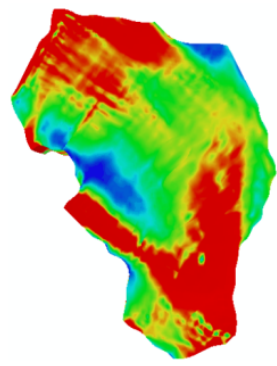

(b)

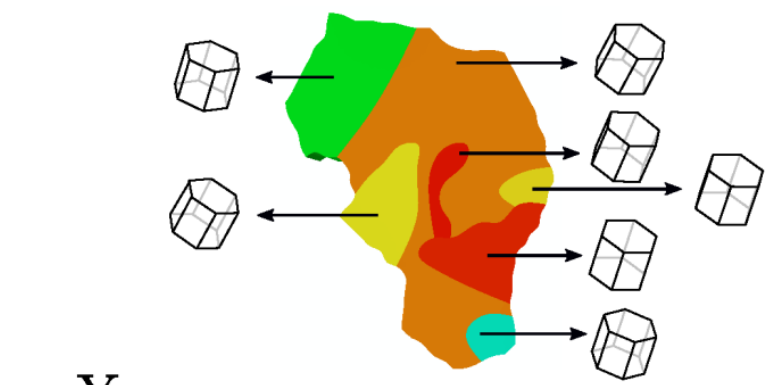

(c)

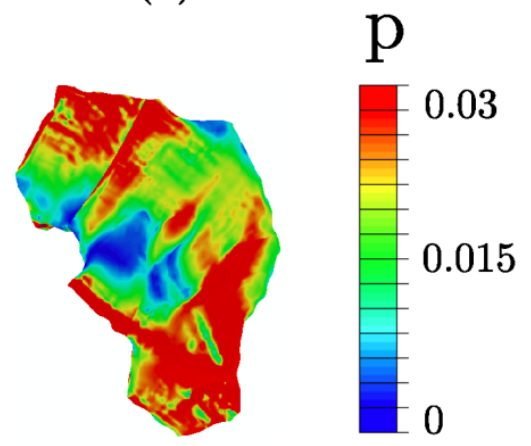

(d)

Figure 17 Effect of initial grain misorientation on local plastic strain fields for grain g2 in Figure 7: illustration of grain g2 without and with misorientation in (a) and (c), respectively; effective plastic strain fields in grain g2 without and with initial misorientation in (b) and (d), respectively.
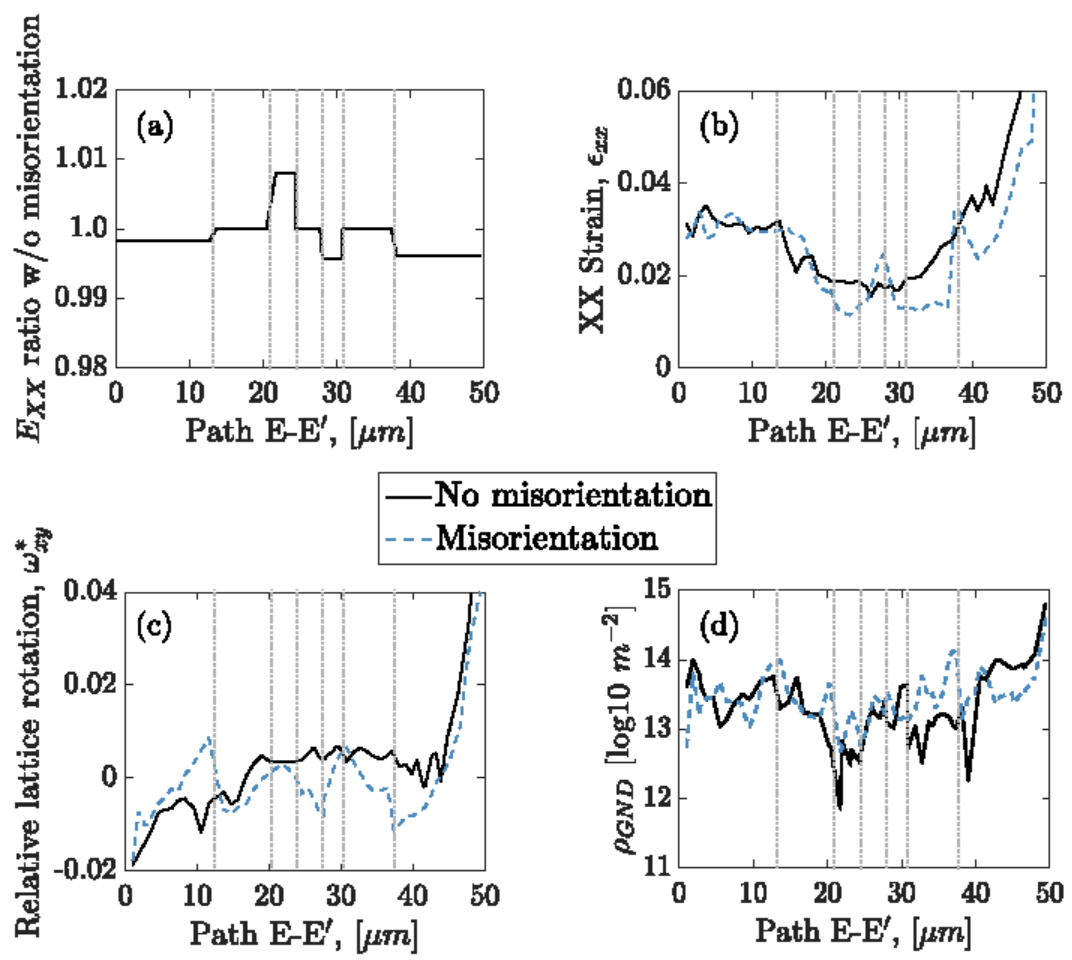

Figure 18 Role of crystal misorientation in micromechanical response in path E-E': (a) tangential modulus ratio, i.e. with misorientation to without misorientation, in loading direction xx; (b) xx strain; (c) relative lattice rotation; (d) GND 
Further examination of the response in grain g2 along path E-E' shown in Figure 16(c), resulting from the differing initial misorientations given in Figure 17(a) and (c), respectively, is given in Figure 18. The grain level misorientations, naturally, change the elastic modulus values, and particularly that component in the loading direction, $\mathrm{E}_{\mathrm{xx}}$. Figure 18(a) shows the spatial variation of modulus $\mathrm{E}_{\mathrm{xx}}$ component shown as the ratio for the misorientated to uniform grain g2. The abrupt change of modulus ratio is clear along path E-E', for which $\mathrm{xx}$ strain and lattice rotation, and GND density at the end of the loading in the CPFE modelling are similarly found to change quite considerably, as a consequence of the differing initial misorientations in grain g2. A similar CPFE computational study carried out for grain g1, where the maximum misorientation after loading is $5^{\circ}$ showed no obvious change of the micromechanical response. These analyses, although limited, suggest that the local mechanical response remains largely unaffected if grain misorientation is less than $5^{\circ}$.

\section{Conclusions}

Quantitative analysis of microscale deformation, slip activation and localization has been conducted in alloy Ti-6Al-4V, using HR-DIC, HR-EBSD and rate-dependent crystal plasticity modelling. The slip activation from HR-DIC measurement and numerical simulation has achieved over $80 \%$ agreement for the primary slip activities in each grain (over 50) in the polycrystal studied. The modelling of 3-D microstructure as columnar grains leads to good agreement (i.e. within $20 \%$ ) in terms of local deformation in about $60 \%$ of the grains. The Schmid factor and slip system activation distributions from HR-DIC measurement and $\mathrm{CP}$ modelling are found to show good agreement. Considering the small deformation in the current case, the apparent Schmid factor calculated using the remote loading direction does not vary very much in local grains. 
The deformation of the sample is accommodated mostly by the activated basal and prismatic slip systems with a Schmid factor over 0.4. In the present study, it has been found more prismatic slips have been activated than basal slips, both from experiment and CP modelling.

Generally good agreement has been achieved in transgranular HR-DIC measurement and CP model strains and lattice rotations in polycrystal deformation. The HR-EBSD GND density distributions are also reasonably reproduced by $\mathrm{CP}$ modelling, though the $\mathrm{CP}$ predicted GND density is found to be consistently lower than that from the HR-EBSD measurement, in keeping with other published results. The role of sub-layer grain texture (from random to extreme) on free-surface slip measurement has also been investigated. A hard subsurface layer of grains will promote the slip accumulation at the grain boundaries of the surface grains.

In this study we showed that it is possible to capture the slip trace in local grain with a size of $10 \mu \mathrm{m}$. The origins of observed single (straight), double (cross) and multiple wavy slip have been explored from the HR-DIC measurements and CP modelling. Wavy slip has been found to result from the interaction of activated slip systems of differing type, but with common slip direction. Finally, initial inter-granular misorientations greater than about $5^{\circ}$ have been shown to influence the subsequent micromechanical grain behaviour including slip, lattice rotation and GND density.

\section{Acknowledgement}

The authors gratefully acknowledge the Engineering \& Physical Science Research Council for funding through HexMat (EP/K034332). Further details of the HexMat grant can be found at http://www.imperial.ac.uk/hexmat. 


\section{References}

Abdolvand, H., Wilkinson, A.J., 2016a. Assessment of residual stress fields at deformation twin tips and the surrounding environments. Acta Materialia 105, 219-231.

Abdolvand, H., Wilkinson, A.J., 2016b. On the effects of reorientation and shear transfer during twin formation: comparison between high resolution electron backscatter diffraction experiments and a crystal plasticity finite element model. International Journal of Plasticity 84, 160-182.

Abdolvand, H., Wright, J., Wilkinson, A.J., 2018. Strong grain neighbour effects in polycrystals. Nature communications $9,171$.

Akhtar, A., 1975. Basal slip and twinning in $\alpha$-titanium single crystals. Metallurgical and Materials Transactions A 6, 1105-1113.

Akhtar, A., Teghtsoonian, E., 1975. Prismatic slip in $\alpha$-titanium single crystals. Metallurgical and Materials Transactions A 6, 2201-2208.

Arsenlis, A., Parks, D., 1999. Crystallographic aspects of geometrically-necessary and statistically-stored dislocation density. Acta materialia 47, 1597-1611.

Ashby, M., 1970. The deformation of plastically non-homogeneous materials. Philos. Mag. $21,399-424$.

Attallah, M., Zabeen, S., Cernik, R., Preuss, M., 2009. Comparative determination of the $\alpha / \beta$ phase fraction in $\alpha+\beta$-titanium alloys using X-ray diffraction and electron microscopy. Materials Characterization 60, 1248-1256.

Bridier, F., Villechaise, P., Mendez, J., 2005. Analysis of the different slip systems activated by tension in a $\alpha / \beta$ titanium alloy in relation with local crystallographic orientation. Acta Materialia 53, 555-567.

Britton, T., Wilkinson, A., 2012. High resolution electron backscatter diffraction measurements of elastic strain variations in the presence of larger lattice rotations. Ultramicroscopy 114, 82-95.

Britton, T.B., Birosca, S., Preuss, M., Wilkinson, A.J., 2010a. Electron backscatter diffraction study of dislocation content of a macrozone in hot-rolled Ti-6Al-4V alloy. Scripta Materialia $62,639-642$.

Britton, T.B., Liang, H., Dunne, F.P.E., Wilkinson, A.J., 2010b. The effect of crystal orientation on the indentation response of commercially pure titanium: experiments and simulations, Proceedings of the Royal Society of London A: Mathematical, Physical and Engineering Sciences. The Royal Society, pp. 695-719.

Britton, T.B., Wilkinson, A.J., 2011. Measurement of residual elastic strain and lattice rotations with high resolution electron backscatter diffraction. Ultramicroscopy 111, 13951404.

Busso, E., Meissonnier, F., O'Dowd, N., 2000. Gradient-dependent deformation of two-phase single crystals. Journal of the Mechanics and Physics of Solids 48, 2333-2361. 
Cermelli, P., Gurtin, M.E., 2001. On the characterization of geometrically necessary dislocations in finite plasticity. Journal of the Mechanics and Physics of Solids 49, 1539-1568.

Clayton, J.D., 2010. Nonlinear mechanics of crystals. Springer Science \& Business Media.

Devincre, B., Hoc, T., Kubin, L., 2008. Dislocation mean free paths and strain hardening of crystals. Science $320,1745-1748$.

Di Gioacchino, F., da Fonseca, J.Q., 2013. Plastic strain mapping with sub-micron resolution using digital image correlation. Experimental Mechanics 53, 743-754.

Di Gioacchino, F., da Fonseca, J.Q., 2015. An experimental study of the polycrystalline plasticity of austenitic stainless steel. International Journal of Plasticity 74, 92-109.

Dunne, F.P.E., Rugg, D., 2008. On the mechanisms of fatigue facet nucleation in titanium alloys. Fatigue Fract. Eng. M. 31, 949-958.

Echlin, M.P., Stinville, J.C., Miller, V.M., Lenthe, W.C., Pollock, T.M., 2016. Incipient slip and long range plastic strain localization in microtextured Ti-6Al-4V titanium. Acta Materialia 114, 164-175.

El-Dasher, B., Adams, B., Rollett, A., 2003. Viewpoint: experimental recovery of geometrically necessary dislocation density in polycrystals. Scripta materialia 48, 141-145.

Franciosi, P., Berveiller, M., Zaoui, A., 1980. Latent hardening in copper and aluminium single crystals. Acta Metallurgica 28, 273-283.

Gao, H., Huang, Y., 2001. Taylor-based nonlocal theory of plasticity. International Journal of Solids and Structures 38, 2615-2637.

Gong, J., Wilkinson, A.J., 2009. Anisotropy in the plastic flow properties of single-crystal $\alpha$ titanium determined from micro-cantilever beams. Acta Mater. 57, 5693-5705.

Gong, J., Wilkinson, A.J., 2011. A microcantilever investigation of size effect, solid-solution strengthening and second-phase strengthening for $<\mathrm{a}>$ prism slip in alpha-Ti. Acta Materialia 59, 5970-5981.

Guan, Y., Chen, B., Zou, J., Britton, T.B., Jiang, J., Dunne, F.P.E., 2017. Crystal plasticity modelling and HR-DIC measurement of slip activation and strain localization in single and oligo-crystal Ni alloys under fatigue. International Journal of Plasticity 88, 70-88.

Guery, A., Hild, F., Latourte, F., Roux, S., 2016. Slip activities in polycrystals determined by coupling DIC measurements with crystal plasticity calculations. International Journal of Plasticity 81, 249-266.

Guo, Y., Abdolvand, H., Britton, T., Wilkinson, A., 2017. Growth of $\left\{112^{-} 2\right\}$ twins in titanium: A combined experimental and modelling investigation of the local state of deformation. Acta Materialia 126, 221-235.

Gurtin, M.E., 2002. A gradient theory of single-crystal viscoplasticity that accounts for geometrically necessary dislocations. Journal of the Mechanics and Physics of Solids 50, 532 . 
Hémery, S., Villechaise, P., 2017. On the influence of ageing on the onset of plastic slip in Ti-6Al-4V at room temperature: Insight on dwell fatigue behavior. Scripta Materialia 130, 157-160.

Jiang, J., Zhang, T., Dunne, F.P.E., Britton, T.B., 2016. Deformation compatibility in a single crystalline Ni superalloy, Proc. R. Soc. A. The Royal Society, p. 20150690.

Kasemer, M., Echlin, M.P., Stinville, J.C., Pollock, T.M., Dawson, P., 2017. On slip initiation in equiaxed $\alpha / \beta$ Ti-6Al-4V. Acta Materialia 136, 288-302.

Li, H., Boehlert, C., Bieler, T., Crimp, M., 2015. Examination of the distribution of the tensile deformation systems in tension and tension-creep of Ti-6Al-4V (wt.\%) at $296 \mathrm{~K}$ and 728 K. Philosophical Magazine 95, 691-729.

Lunt, D., Busolo, T., Xu, X., da Fonseca, J.Q., Preuss, M., 2017a. Effect of nanoscale $\alpha 2$ precipitation on strain localisation in a two-phase Ti-alloy. Acta Materialia 129, 72-82.

Lunt, D., da Fonseca, J.Q., Rugg, D., Preuss, M., 2017b. Microscopic strain localisation in Ti-6Al-4V during uniaxial tensile loading. Materials Science and Engineering: A 680, 444453.

Lunt, D., Xu, X., Busolo, T., da Fonseca, J.Q., Preuss, M., 2018. Quantification of strain localisation in a bimodal two-phase titanium alloy. Scripta Materialia 145, 45-49.

Lütjering, G., Williams, J.C., 2007. Titanium. Springer, Berlin Heidelberg.

Miller, M., Dawson, P., 2014. Understanding local deformation in metallic polycrystals using high energy X-rays and finite elements. Current Opinion in Solid State and Materials Science 18, 286-299.

Nye, J., 1953. Some geometrical relations in dislocated crystals. Acta metallurgica 1, 153-162.

St-Pierre, L., Héripré, E., Dexet, M., Crépin, J., Bertolino, G., Bilger, N., 2008. 3D

simulations of microstructure and comparison with experimental microstructure coming from OIM analysis. International Journal of Plasticity 24, 1516-1532.

Steinmann, P., 2015. Geometrical foundations of continuum mechanics. Springer.

Stinville, J., Vanderesse, N., Bridier, F., Bocher, P., Pollock, T., 2015. High resolution mapping of strain localization near twin boundaries in a nickel-based superalloy. Acta Materialia 98, 29-42.

Sun, S., Adams, B., King, W., 2000. Observations of lattice curvature near the interface of a deformed aluminium bicrystal. Philosophical Magazine A 80, 9-25.

Tasan, C.C., Hoefnagels, J.P., Diehl, M., Yan, D., Roters, F., Raabe, D., 2014. Strain localization and damage in dual phase steels investigated by coupled in-situ deformation experiments and crystal plasticity simulations. International Journal of Plasticity 63, 198-210.

Vendroux, G., Knauss, W.G., 1998. Submicron deformation field measurements: Part 2. Improved digital image correlation. Experimental Mechanics 38, 86-92. 
Wan, V., Cuddihy, M., Jiang, J., MacLachlan, D., Dunne, F.P.E., 2016. An HR-EBSD and computational crystal plasticity investigation of microstructural stress distributions and fatigue hotspots in polycrystalline copper. Acta Materialia 115, 45-57.

Wilkinson, A.J., Meaden, G., Dingley, D.J., 2006a. High-resolution elastic strain measurement from electron backscatter diffraction patterns: new levels of sensitivity. Ultramicroscopy 106, 307-313.

Wilkinson, A.J., Meaden, G., Dingley, D.J., 2006b. High resolution mapping of strains and rotations using electron backscatter diffraction. Materials Science and Technology 22, 12711278.

Wilkinson, A.J., Randman, D., 2010. Determination of elastic strain fields and geometrically necessary dislocation distributions near nanoindents using electron back scatter diffraction. Philosophical magazine 90, 1159-1177.

Williams, J.C., Baggerly, R.G., Paton, N.E., 2002. Deformation behavior of HCP Ti-Al alloy single crystals. Metallurgical and Materials Transactions A 33, 837-850.

Yang, Y., Wang, L., Bieler, T., Eisenlohr, P., Crimp, M., 2011. Quantitative atomic force microscopy characterization and crystal plasticity finite element modeling of heterogeneous deformation in commercial purity titanium. Metallurgical and Materials Transactions A 42, 636-644.

Zeghadi, A., N'guyen, F., Forest, S., Gourgues, A.-F., Bouaziz, O., 2007. Ensemble averaging stress-strain fields in polycrystalline aggregates with a constrained surface microstructurePart 1: Anisotropic elastic behaviour. Philosophical Magazine 87, 1401-1424.

Zhang, C., Li, H., Eisenlohr, P., Liu, W., Boehlert, C., Crimp, M., Bieler, T., 2015a. Effect of realistic 3D microstructure in crystal plasticity finite element analysis of polycrystalline Ti5Al-2.5 Sn. International Journal of Plasticity 69, 21-35.

Zhang, T., Collins, D.M., Dunne, F.P.E., Shollock, B.A., 2014. Crystal plasticity and highresolution electron backscatter diffraction analysis of full-field polycrystal Ni superalloy strains and rotations under thermal loading. Acta Materialia 80, 25-38.

Zhang, T., Jiang, J., Shollock, B.A., Britton, T.B., Dunne, F.P.E., 2015b. Slip localization and fatigue crack nucleation near a non-metallic inclusion in polycrystalline nickel-based superalloy. Materials Science and Engineering: A 641, 328-339.

Zhang, Z., Cuddihy, M.A., Dunne, F.P.E., 2015c. On rate-dependent polycrystal deformation: the temperature sensitivity of cold dwell fatigue. Proc. R. Soc. A 471, 20150214.

Zhang, Z., Dunne, F.P.E., 2017. Microstructural heterogeneity in rate-dependent plasticity of multiphase titanium alloys. Journal of the Mechanics and Physics of Solids 103, 199-220.

Zhang, Z., Jun, T.-S., Britton, T.B., Dunne, F.P.E., 2016a. Determination of Ti-6242 $\alpha$ and $\beta$ slip properties using micro-pillar test and computational crystal plasticity. Journal of the Mechanics and Physics of Solids 95, 393-410.

Zhang, Z., Jun, T.-S., Britton, T.B., Dunne, F.P.E., 2016b. Intrinsic anisotropy of strain rate sensitivity in single crystal alpha titanium. Acta Materialia 118, 317-330. 